\title{
The Dynamics of Vortex Rossby Waves and Secondary Eyewall Development in Hurricane Matthew (2016): New Insights from Radar Measurements
}

\author{
STEPHEN R. GUIMOND \\ University of Maryland, Baltimore County, Baltimore, and NASA Goddard Space Flight Center, Greenbelt, Maryland \\ PAUL D. REASOR \\ NOAA/Hurricane Research Division, Miami, Florida \\ Gerald M. HeymSFiEld AND MATTHEw M. MCLindeN \\ NASA Goddard Space Flight Center, Greenbelt, Maryland
}

(Manuscript received 12 October 2019, in final form 29 April 2020)

\begin{abstract}
The structure of vortex Rossby waves (VRWs) and their role in the development of a secondary eyewall in Hurricane Matthew (2016) is examined from observations taken during the NOAA Sensing Hazards with Operational Unmanned Technology (SHOUT) field experiment. Radar measurements from ground-based and airborne systems, with a focus on the NASA High-Altitude Imaging Wind and Rain Airborne Profiler (HIWRAP) instrument on the Global Hawk aircraft, revealed the presence of $\sim 12-15-\mathrm{km}$-wavelength spiral bands breaking from the inner-core eyewall in the downshear-right quadrant. The vorticity characteristics and calculations of the intrinsic phase speeds of the bands are shown to be consistent with sheared VRWs. A new angular momentum budget methodology is presented that allows an understanding of the secondary eyewall development process with narrow-swath radar measurements. Filtering of the governing equations enables explicit insight into the nonlinear dynamics of scale interactions and the role of the VRWs in the storm structure change. The results indicate that the large-scale (scales $>15 \mathrm{~km}$ ) vertical flux convergence of angular momentum associated with the VRWs dominates the time tendency with smaller effects from the radial flux term. The small-scale (scales $\leq 15 \mathrm{~km}$ ) vertical term produces weak, but nonnegligible nonlinear forcing of the large scales primarily through the Reynolds and cross-stress components. The projection of the wave kinematics onto the low-wavenumber ( 0 and 1$)$ fields appears to be the more significant dynamic process. Flight-level observations show secondary peaks in tangential winds in the radial region where the VRW forcing signatures are active, connecting them with the secondary eyewall formation process.
\end{abstract}

\section{Introduction}

Spiral bands of various scales have been observed in the low to middle levels $(\sim 5 \mathrm{~km}$ and below) of hurricanes for some time, and theories for their origin, characteristics, and impacts on storm intensity and structure change have been examined in detail. The oscillatory nature of the observed fields, such as radar reflectivity (e.g., Gall et al. 1998), suggests that a wave phenomenon may govern the structure and characteristics of the banded features. Early studies postulated that hurricane spiral bands were initiated by inertia-buoyancy

Corresponding author: Stephen R. Guimond, sguimond@umbc.edu waves either forced by diabatic heating and propagating outward or originating in the environment and propagating inward (Kurihara 1976; Willoughby 1978). However, Guinn and Schubert (1993) used a shallow water model to show that the vast majority of energy in bands projects onto the rotational modes with little influence from gravitational modes associated with inertiabuoyancy waves. The authors proposed that inner-core bands are produced from the nonlinear breaking of potential vorticity (PV) waves that propagate along the basic-state radial PV gradient and are usually termed vortex Rossby waves (VRWs; Macdonald 1968).

After periods of significant convective activity associated with the hurricane intensification process, 
the storm enters a mature stage where the eyewall typically displays a ring of enhanced PV or vorticity with oppositely signed radial gradients on the inner and outer edge. Schubert et al. (1999) showed that such a vortex structure supports counterpropagating VRWs that can phase lock and grow to finite amplitude leading to the breakdown of the eyewall into coherent turbulent structures (mesovortices) and the outward propagation of vorticity filaments. These outward-propagating vorticity filaments may be similar to those discussed by Guinn and Schubert (1993).

Strong, pulsing convective activity, such as convective bursts, is typically sporadic and weaker during the mature stage (e.g., Guimond et al. 2010; Abarca et al. 2011) and secondary eyewalls have been observed to form (e.g., Willoughby et al. 1982; Black and Willoughby 1992; Willoughby and Black 1996; Bell et al. 2012; Didlake et al. 2017). The current study focuses on the mature and weakening stage of the hurricane life cycle, but this does not mean that the system is devoid of pulsing convection. In addition to the vortex instability mechanisms for VRW generation described above, convectively forced VRWs are also possible (e.g., Montgomery and Enagonio 1998).

Montgomery and Kallenbach (1997, hereafter MK97) studied the dynamics of spiral bands in hurricane-like vortices and developed a linear, barotropic theory for VRWs, which describes their propagation characteristics and interaction with the azimuthal-mean vortex. Trailing spiral VRWs were shown to propagate radially outward and increase their radial wavenumber due to sustained radial shearing associated with the differential rotation of the vortex. MK97 suggested that inner-core convective bands often seen in satellite and radar imagery could be initiated through the vertical motions associated with VRWs. As the VRWs shear to finer radial scales and slow their outward propagation, they stagnate and interact with the mean flow through eddy momentum flux convergence or, equivalently, eddy vorticity fluxes, leading to spin up (spin down) approximately radially inward (outward) of the stagnation radius. Möller and Montgomery (2000) subsequently demonstrated that if the VRWs can sustain this forcing over an extended period of time, significant modifications of the mean vortex structure can occur.

One such structural impact of VRWs proposed by MK97, relevant to the present study, is the development of secondary eyewalls. The role of VRWs in secondary eyewall formation has been studied primarily through idealized and "full-physics" numerical models (e.g., Kuo et al. 2008; Terwey and Montgomery 2008; Qiu et al. 2010; Abarca and Corbosiero 2011). Simple models show that the axisymmetrization of small-scale vorticity anomalies in the outer regions of the vortex core can produce a secondary peak in tangential wind (Kuo et al. 2008). Idealized, full-physics modeling studies illustrate that convectively generated vorticity anomalies in the outer-core region, where a broad envelope of background vorticity is found, can axisymmetrize and form a secondary eyewall (Terwey and Montgomery 2008; Qiu et al. 2010). However, it is not clear from these studies what role the VRWs are playing in the secondary eyewall evolution process. Are they only acting to expand the outer envelope of vorticity, which facilitates the axisymmetrization of convective-scale vorticity anomalies, or do they play a direct role in the outer-core spinup? Qiu et al. (2010) examined some of these issues in a modeling study and concluded that most of the secondary eyewall formation can be explained by the axisymmetrization of vorticity perturbations in an outer rainband. The VRWs were viewed as indirectly impacting the secondary eyewall formation, by radially expanding the region of large-scale vorticity that enables the axisymmetrization of small-scale anomalies.

Observational investigations into hurricane innercore spiral bands and VRW dynamics are much more limited relative to modeling based papers, but they are a key component in developing comprehensive knowledge of hurricane physics. Reasor et al. (2000) used wind fields derived from airborne radar data to characterize the asymmetric dynamics of a weakening storm. The authors found evidence for low-wavenumber (dominated by azimuthal wavenumber two) vorticity asymmetries within the eyewall that resembled discrete VRWs and spiral bands of vorticity outside the eyewall with radial wavelengths of 5-10 km. The authors did not compute the propagation of the observed bands to verify the existence of VRWs. Instead, using the VRW theory of MK97, the authors determined that a secondary maximum in mean vorticity radially outside the eyewall was consistent with the outward propagation, stagnation, and mean-flow interaction of spiral bands having the observed structure.

Corbosiero et al. (2006) analyzed ground-based radar reflectivity measurements in an intensifying storm and determined that the azimuthal phase speeds of banded features were consistent with the VRW theory; an additional piece of evidence identifying VRWs that is not easily assessed using airborne data. Didlake and Houze (2011) examined one snapshot of airborne radar data in Hurricane Rita (2005) and showed oscillations in the perturbation vorticity field in the primary and secondary eyewall during a concentric eyewall cycle of the storm. The banded vorticity features had wavelengths of 6 $10 \mathrm{~km}$ and were coupled to the convective field. The authors suggested the perturbations were consistent 
with VRWs active in the storm, but no calculations were performed to examine this idea more closely.

Recently, Guimond et al. (2018) presented new radar measurements of finescale bands in the boundary layer of Hurricane Rita (2005) with radial wavelengths of $\sim 2 \mathrm{~km}$, depths from the ocean surface up to flight level $(\sim 1.5-\mathrm{km}$ height $)$ and horizontal wind speed perturbations of $10-20 \mathrm{~m} \mathrm{~s}^{-1}$. Many of the turbulent eddies had circulation signatures with positive radial and vertical momentum fluxes, which is different than the prevailing view of boundary layer rolls (e.g., Morrison et al. 2005). Given this information, the authors suggested that the bands may be connected to VRWs. In a new study of secondary eyewall formation in Hurricane Irma (2017), Fischer et al. (2020) showed overlapping spiral bands of reflectivity and vorticity derived from airborne radar measurements. In addition to retrogression of the bands in a manner consistent with VRWs, theoretical estimates of VRW stagnation radii coincided with the regions of secondary eyewall formation. The authors did not quantify the role of the bands in the observed secondary eyewall development.

It is clear that more detailed measurements of banded features, such as VRWs, in hurricanes are needed to characterize their structure and, more importantly, estimate their effects on the storm intensity and structure change. The purpose of this paper is to analyze a set of new, high-resolution measurements of spiral bands in hurricanes from a unique platform, understand their structure and estimate their impacts in the secondary eyewall formation process. In this paper, we define a secondary eyewall as a clear secondary tangential wind maximum located radially outward from the main eyewall.

\section{Data and processing}

\section{a. HIWRAP}

The High-Altitude Imaging Wind and Rain Airborne Profiler (HIWRAP) is a dual-frequency ( $\mathrm{Ku}$ and $\mathrm{Ka}$ band), downward-pointing, and conically scanning (16 revolutions per minute; rpm) airborne Doppler radar. The HIWRAP has two beams ( $\sim 30^{\circ}$ and $40^{\circ}$ tilt angles), $75-\mathrm{m}$ range resolution, and operates on the NASA Global Hawk (GH) unmanned aircraft at $\sim 18-19-\mathrm{km}$ (60-62 kft) altitude. The GH aircraft has an airspeed of $\sim 160 \mathrm{~m} \mathrm{~s}^{-1}$, which provides $\sim 600$-m along-track sampling for HIWRAP. Additional details on the HIWRAP instrument specifications can be found in Li et al. (2016). The GH aircraft has long endurance capability with flights up to $\sim 24 \mathrm{~h}$, which is of significant value for hurricane research where data sources are often very limited. Satellite measurements can provide valuable large-scale overviews of storms, but the observational capabilities, including spatial and temporal sampling, is not optimal for detailed dynamic studies.

Three-dimensional wind vector retrievals covering the HIWRAP sampling volume are performed with the three-dimensional variational (3DVAR) algorithm described in Guimond et al. (2014, 2018). In this study, the winds are computed on a track-relative retrieval grid with $1-\mathrm{km}$ horizontal and $0.25-\mathrm{km}$ vertical spacing. No Laplacian filtering was used in the minimization of the cost function, but a two-point running-mean filter was applied to the retrievals in postprocessing to remove gridscale noise. The HIWRAP antenna main- and sidelobes interact strongly with the ocean surface and obscure the precipitation signal below $\sim 1-\mathrm{km}$ altitude for both the inner and outer beams. Therefore, the wind retrievals start at $1-\mathrm{km}$ height for the present study.

More details on the wind vector retrieval process can be found in Guimond et al. (2014, 2016, 2018), including detailed error characterization. Validation of the HIWRAP wind retrievals with dropsonde data was performed for this study, but they are not shown for brevity. The HIWRAP retrievals validated well within the scope of the point measurements made by the dropsondes (the radar winds represent a larger, filtered scale) and they provide confidence moving forward with the science. Also note that storm center estimates are provided by the Air Force and NOAA aircraft center fixes interpolated to the HIWRAP overpass times. The storm motion vector, calculated from aircraft center fixes, averaged over the aircraft-sampling period was removed from the HIWRAP derived horizontal winds for analysis. The magnitude of the storm motion vector was $4.61 \mathrm{~m} \mathrm{~s}^{-1}$ and pointed toward the north-northwest.

\section{b. WSR-88Ds}

The WSR-88D ground-based network is a well-known S-band radar system with a $0.96^{\circ}$ one-way, 3 -dB beamwidth and a range resolution of $250 \mathrm{~m}$. In this work, standard level 2 data products (e.g., gridded reflectivity) are used from the Melbourne and Jacksonville, Florida, radars to characterize both the larger-scale storm structure and smaller-scale details in both space and time. The grid spacing of the data is $1 \mathrm{~km}$ for reflectivity with $1^{\circ}$ azimuthal sampling.

\section{Overview of Hurricane Matthew}

During the summer of 2016, NOAA conducted the Sensing Hazards with Operational Unmanned Technology (SHOUT) field experiment to study the impact of the $\mathrm{GH}$ aircraft and a suite of in situ and remote sensing 


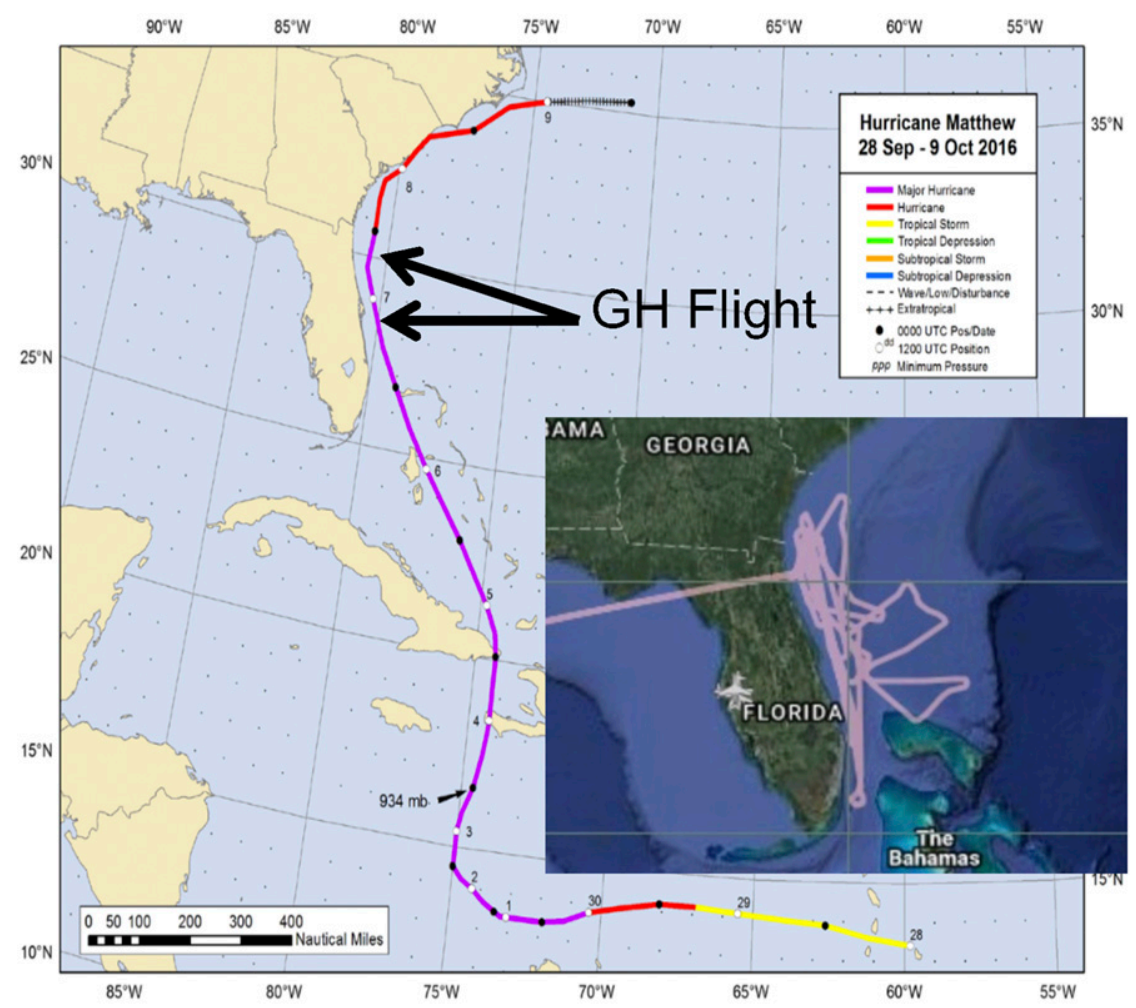

FIG. 1. Best track of Hurricane Matthew (2016) and identification of the SHOUT flight on 7 Oct. The main figure is courtesy of the National Hurricane Center (NHC). The inset shows the track of the Global Hawk (GH) aircraft during 1000-2000 UTC 7 Oct.

instruments on the forecasting of hurricane track, intensity, and structure. In early October of 2016, Hurricane Matthew presented a quality target for $\mathrm{GH}$ flights to study the SHOUT goals for a storm that posed a potentially significant operational challenge. Matthew became a named system on 28 September in the Caribbean Sea and shortly after attained hurricane status including a period of extremely rapid intensification [75-kt $\left(1 \mathrm{kt} \approx 0.51 \mathrm{~m} \mathrm{~s}^{-1}\right)$ increase in maximum sustained winds in $24 \mathrm{~h}$; Stewart 2017].

The focus of this paper is on the later portion of Matthew's life cycle, from $\sim 6$ to 8 October when the majority of the SHOUT flights occurred. Figure 1 shows the best track of Matthew along with labeling that indicates the time period of the GH sampling $\sim 1000$ 2000 UTC 7 October and an inset that shows the flight track. Figure 2 shows the intensity time series of the storm. After grazing Haiti and Cuba on 4-5 October, Matthew reintensified on 6 October and then began a gradual weakening trend due to an eyewall replacement cycle and a hostile environment with increasing vertical wind shear from an approaching trough (Stewart 2017). The operational Statistical Hurricane Intensity Prediction Scheme (SHIPS) indicated that the 850-200-hPa, large-scale vertical shear surrounding Matthew during the GH sampling period was $\sim 9 \mathrm{~m} \mathrm{~s}^{-1}$ toward $\sim 45^{\circ}$ (southwesterly). The SHOUT observations on 7 October occurred during this slow, but steady weakening period (Fig. 2). During this observation period, a new secondary eyewall (defined by secondary tangential wind maxima) was observed in both flight level and HIWRAP data.

\section{Vortex Rossby wave remote sensing observations}

\section{a. Ground-based radar evolution}

Animations of base scan $\left(0.50^{\circ}\right)$ WSR-88D data from Melbourne indicate that spiral bands of reflectivity in the inner core of Matthew were apparent on 7 October 2016 during a concentric eyewall stage of the storm. The system was moving past the radar on this day and the storm center was located $\sim 100 \mathrm{~km}$ from the Melbourne WSR-88D during the time period of focus for the bulk of the analysis in this section (e.g., 1200-1500 UTC). During this time period, the height of the radar beam in the northern/northeastern sections of the storm at 


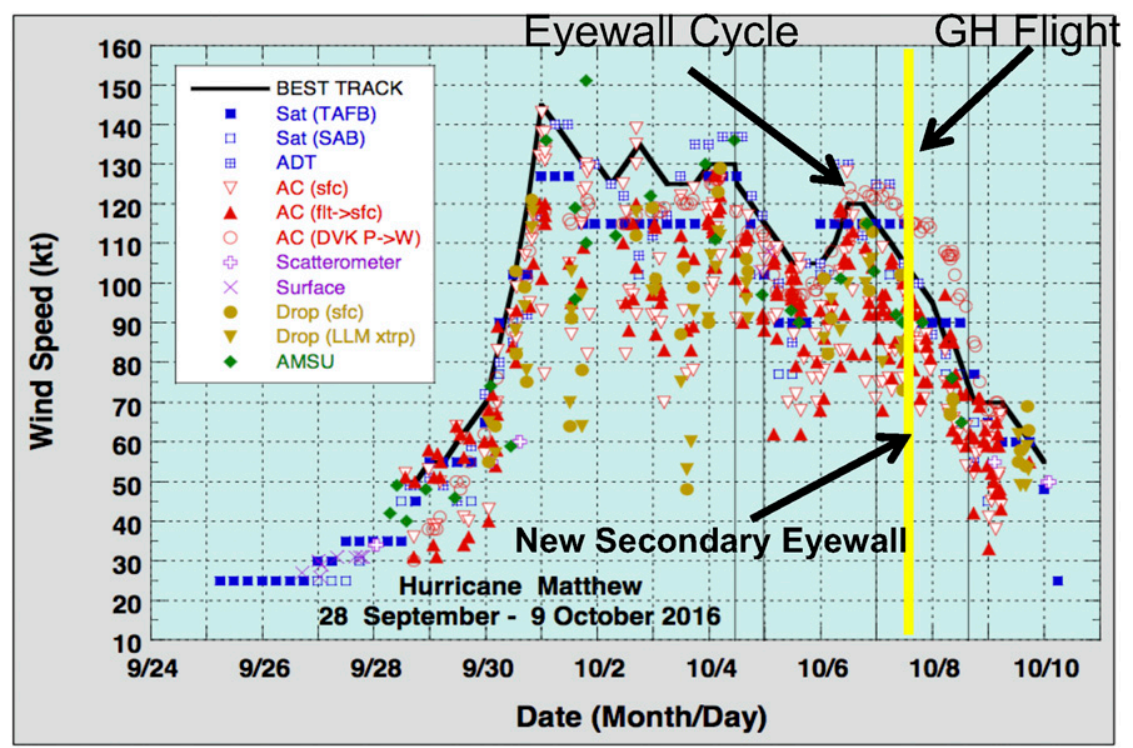

FIG. 2. Intensity time series of Hurricane Matthew (2016). The main figure is courtesy of the NHC. The beginning of an eyewall replacement cycle and the GH flight on 7 Oct (yellow line) is marked. A new secondary eyewall observed during the GH flight time period is also marked.

$\sim 50 \mathrm{~km}$ radius is $\sim 1.50 \mathrm{~km}$ with a footprint diameter (aligned roughly in the vertical direction) of $\sim 2.5 \mathrm{~km}$ based on the $0.96^{\circ}$ beamwidth. As will be shown below, these measurements resolve the spiral bands in the storm quite well. Observations from the Jacksonville WSR-88D were also analyzed and they showed similar results to the Melbourne data.

Figure 3 shows four snapshots of the reflectivity field from the Melbourne WSR-88D animation that depict the typical evolution of the banded features. In Fig. 3a (1047 UTC), thin bands of enhanced reflectivity are breaking off the main eyewall (see black arrows) in the downshear-right quadrant of the storm. These bands have a radial wavelength of $\sim 12 \mathrm{~km}$ based on close visual inspection. The main eyewall feature in Fig. 3a is really the outer eyewall $(\sim 50-\mathrm{km}$ radius $)$ in an eyewall replacement cycle that has intensified and contracted, leading to the dissipation of the inner eyewall $(\sim 25-\mathrm{km}$ radius). The inner eyewall can be seen in Fig. $3 \mathrm{a}$ as a wavenumber one reflectivity feature inside the main eyewall. About one hour later at 1155 UTC (Fig. 3b), the thin bands of reflectivity have separated and moved both radially and azimuthally, which continues at 1240 UTC (Fig. 3c) and 1348 UTC (Fig. 3d). At the outer radii, the bands have a radial wavelength of $\sim 15 \mathrm{~km}$. Figures $3 \mathrm{c}$ and $3 \mathrm{~d}$ indicate that the spiral bands should project strongly onto azimuthal wavenumbers zero and one given the large azimuthal distances they cover (see dotted black lines for examples). The band reflectivity signature is more difficult to observe on the southern side of the storm due to the upshear suppression of convection.

The spiral reflectivity bands identified in Fig. 3 are connected with vorticity bands, which assists in classifying the bands as VRWs given the intrinsic vortical nature of these features (Guinn and Schubert 1993; MK97). This connection is illustrated with a composite of two HIWRAP swaths (storm center crossings at 1811 and 1900 UTC) averaged between 1- and 1.5-km height shown in Fig. 4. Note that there are multiple, similar instances of the band evolution described in Fig. 3 and the HIWRAP observations characterize the structure of a representative occurrence of this evolution. The reflectivity field in Fig. 4a shows spiral bands breaking from the main eyewall in the downshear-right quadrant of the storm and wrapping around to the north of the center (see dotted gray curves), which is characteristic of the evolution shown in Fig. 3. Perturbation wind vectors, defined by removing the HIWRAPcomputed azimuthal mean wind from the total wind, are also shown in Fig. 4a and reveal strong outflow of $\sim 20 \mathrm{~m} \mathrm{~s}^{-1}$ where the bands are breaking from the main eyewall. These same spiral bands identified with dotted gray curves are collocated with positive vorticity bands in Fig. 4b. While the vorticity bands have several smallscale perturbations that arise with high-resolution radar measurements in a complicated flow field, they are largely horizontally coherent features.

Hovmöller diagrams were created to track the bands and calculate their propagation characteristics and they 

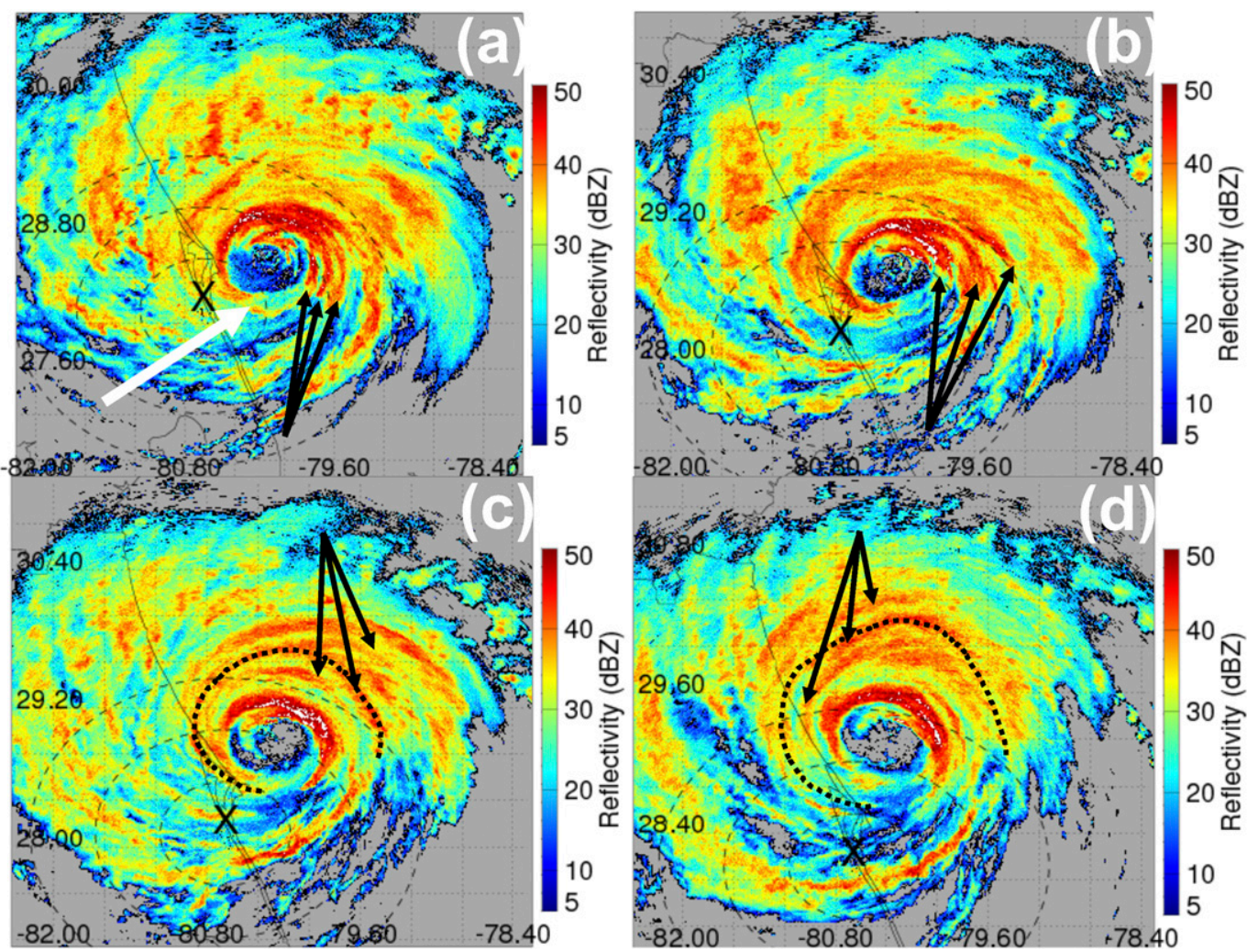

FIG. 3. A sequence of base-scan $\left(0.50^{\circ}\right)$ WSR-88D (Melbourne) reflectivity images of Hurricane Matthew (2016) that cover portions of a Global Hawk research flight into the storm. The times shown are (a) 1047, (b) 1155, (c) 1240, and (d) 1348 UTC 7 Oct 2016. The white arrow in (a) denotes the environmental vertical wind shear vector valid over the time interval. The $\times$ symbol represents the location of the radar. The black arrows in each panel highlight banded reflectivity structures as they propagate around the storm. The dotted black curves in (c) and (d) highlight the azimuthal extent of representative bands.

are shown in Fig. 5. Note that the specific bands labeled in Fig. 5 are representative of the broader time period under investigation. Figure 5a shows an azimuth versus time plot of the radar reflectivity at $50-\mathrm{km}$ radius on 7 October 2016 from the Melbourne WSR-88D. Between 0600 and 2000 UTC, bands (see dashed arrows for examples) of reflectivity (40-45 dBZ) can be seen extending from $\sim 120^{\circ}$ (southeast) to $0^{\circ}$ (north), wrapping around to the northwest and dissipating on the southern side of the storm. The radius versus time plot looking northwest in Fig. 5b shows bands propagating from the main, outer eyewall ( $\sim 50-\mathrm{km}$ radius) out to $\sim 100-\mathrm{km}$ radius at early times (0600-0900 UTC). At later times (1200-1500 UTC) bands (see dashed arrows for examples) move out to $\sim 125-\mathrm{km}$ radius where they show some signs of stagnation. Both attenuation and the coarse resolution of the radar beam at $125-\mathrm{km}$ radius from the storm center during the 1200-1500 UTC time period, however, make definitive statements regarding propagation characteristics a challenge. The inner eyewall is discernable in Fig. $5 \mathrm{~b}$ as elevated reflectivity at the $\sim 25-\mathrm{km}$ radius. Over time, the outer/main eyewall contracts from a radius of $\sim 50$ to $\sim 35 \mathrm{~km}$, while the inner eyewall dissipates and/or merges with the outer/main eyewall at $\sim 1500$ UTC.

The azimuthal phase speeds $C_{p \lambda}$ of the bands identified in Fig. 5a were calculated by estimating the slope of the lines of constant phase over an azimuthal sector (see black arrows in Fig. 5a). Using data at the 50-km radius, the average azimuthal phase speed of the bands was $24.5 \mathrm{~m} \mathrm{~s}^{-1}$. This value reflects azimuthal advection by the Earth-relative flow (largely a superposition of the symmetric vortex and environmental flow) and potential intrinsic propagation of the bands. Quantification of intrinsic propagation is of interest here and HIWRAP data collocated with some of the WSR-88D scans in the azimuthal sectors shown by the black arrows in Fig. 5a allows an estimate of the intrinsic phase speeds.

The azimuthal flow advecting the bands is approximated by the local azimuthal-mean tangential wind, as done in previous observational studies (e.g., Reasor et al. 2000; Corbosiero et al. 2006). The azimuthal-mean 

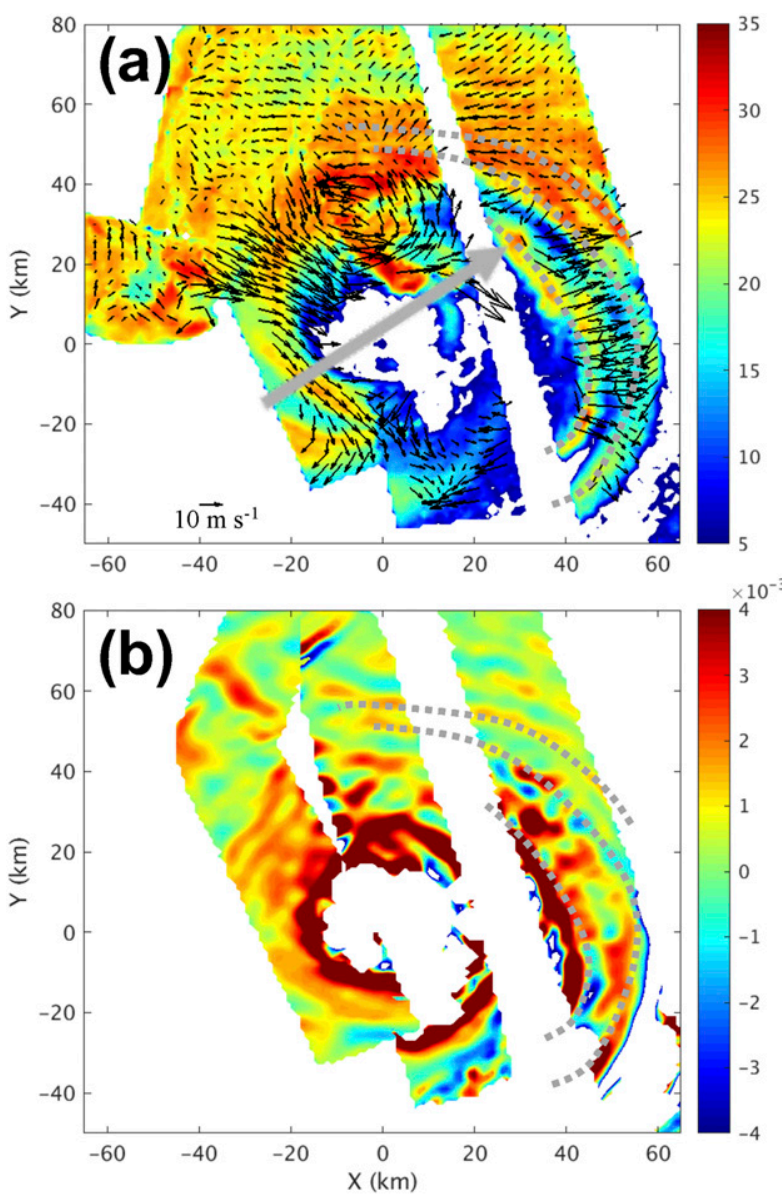

FIG. 4. Composite of HIWRAP data for two overpasses (center crossings at 1811 and 1900 UTC 7 Oct) averaged between 1- and 1.5-km height: (a) radar reflectivity $(\mathrm{dBZ})$ and perturbation wind vectors $\left(\mathrm{m} \mathrm{s}^{-1}\right)$ and (b) vertical vorticity $\left(\mathrm{s}^{-1}\right)$. The large gray arrow in (a) represents the vertical wind shear vector. The dotted gray curves in (a) and (b) denote VRW spiral bands.

tangential wind speed $V_{t}$ is estimated from a composite of HIWRAP data at $1-1.5-\mathrm{km}$ height (see Fig. 4a). Oscillations associated with the bands are present in the HIWRAP-defined azimuthal mean tangential wind profile so a radial filter with a window of $12 \mathrm{~km}$ (radial wavelength of bands at $50-\mathrm{km}$ radius) is applied to the profile. The azimuthal-mean tangential wind speed is approximately $39 \mathrm{~m} \mathrm{~s}^{-1}$ at the location of the bands near $50-\mathrm{km}$ radius. The intrinsic azimuthal phase speed $\left(C_{p \lambda}-V_{t}\right)$ at $50-\mathrm{km}$ radius is then $\sim-14.5 \mathrm{~m} \mathrm{~s}^{-1}$.

The theoretically derived azimuthal phase speed at $50-\mathrm{km}$ radius was also estimated using the barotropic dispersion relation in MK97 with inputs for the various parameters provided by a combination of WSR-88D and HIWRAP data. Using a reference radius of $50 \mathrm{~km}$, radial wavelength of $12 \mathrm{~km}$ and estimated radial vorticity gradient $\left(-1.05 \times 10^{-7} \mathrm{~m}^{-1} \mathrm{~s}^{-1}\right)$, the theoretical intrinsic

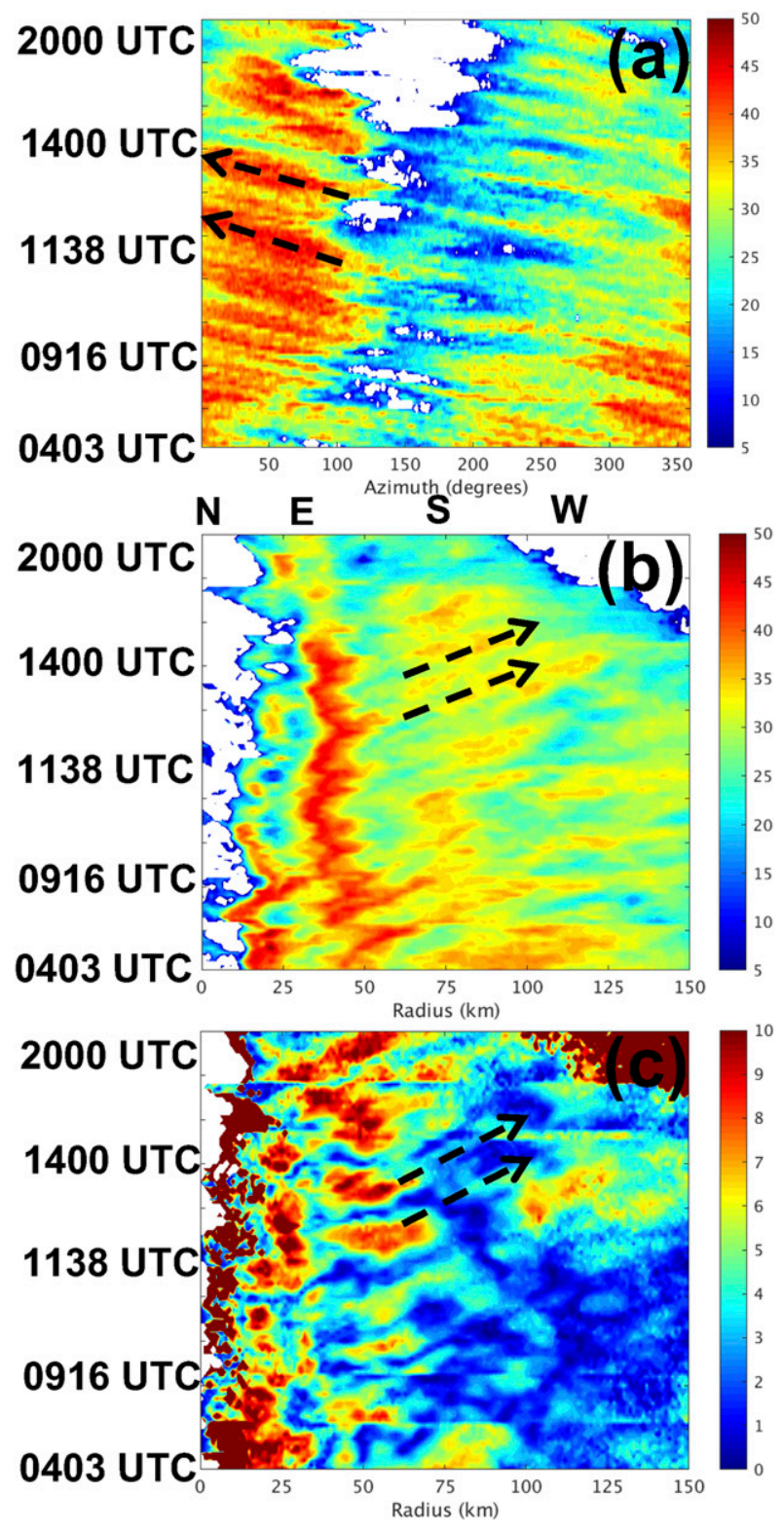

FIG. 5. Hovmöller diagrams of the reflectivity field from the WSR-88D in Melbourne on 7 Oct 2016: (a) azimuth vs time at a radius of $50 \mathrm{~km}$, (b) radius vs time averaged over a $10^{\circ}$ azimuthal sector (looking northwest), and (c) the complex magnitude of the wavenumber- 2 field. The dashed arrows highlight representative bands being tracked in the storm.

azimuthal phase speed for the wavenumber- 1 component relative to the symmetric tangential flow is $\sim-14.3 \mathrm{~m} \mathrm{~s}^{-1}$. This value is close to the measured intrinsic azimuthal phase speed of $\sim-14.5 \mathrm{~m} \mathrm{~s}^{-1}$. It should be acknowledged that there are inherent uncertainties associated with estimating the radial scale of the VRWs and the azimuthal-mean vortex parameters. Additionally, approximating the actual advecting flow of the bands by 
the azimuthal-mean tangential wind (especially in highly asymmetric tropical cyclone flows) introduces some error into the estimate of the measured intrinsic propagation speed. Despite these uncertainties, the comparison described above indicates quite reasonable agreement between the measured intrinsic azimuthal phase speeds and those predicted by VRW theory.

The radial speeds of the bands were estimated following Moon and Nolan (2015) by tracking features in a plot of the complex magnitude of the low-wavenumber $(n>1)$ radar reflectivity. To illustrate the actual radial propagation of features the magnitude is used to remove the apparent radial propagation, or "pinwheel" effect, due to the tangential advection of spiral band features.

Animation of the wavenumber-2 component of reflectivity (not shown) indicated outward propagation of spiral band features related to those shown in the full field (Fig. 3). Figure $5 \mathrm{c}$ shows the complex magnitude of the wavenumber-2 component of radar reflectivity as a function of radius and time. A series of the reflectivity bands evident in the animation can also be tracked in Fig. 5c, starting at $\sim 50-\mathrm{km}$ radius and moving out to $\sim 75-100-\mathrm{km}$ radius. Two of these bands are highlighted with dashed arrows (consistent with those shown in Fig. 5b) and their slopes provide an estimate of the radial speeds yielding a mean outward value of $\sim 8.50 \mathrm{~m} \mathrm{~s}^{-1}$. For context, the azimuthal-mean radial flow at $\sim 3-\mathrm{km}$ height was estimated using Air Force flight-level data distributed over time (1209-1542 UTC) and azimuth revealing average values of $\sim-3.5 \mathrm{~m} \mathrm{~s}^{-1}$ in the radial zone where the spiral bands are active.

The azimuthal and radial speed calculations above indicate that the observed spiral bands in Hurricane Matthew during this time period propagate against the mean tangential flow of the vortex and move radially outward against the mean radial inflow. In addition, the azimuthal phase speeds are in good agreement with barotropic VRW theory. Last, as described above in HIWRAP data (Fig. 4), the spiral bands observed here in the ground-based measurements are largely correlated with positive vorticity bands identified in the airborne data. Further evidence of correlated bands of reflectivity and vorticity are shown later in Figs. 14a and $14 \mathrm{~b}$.

Taken together, the characteristics described above are consistent with trailing spiral VRWs described in the introduction (MK97) and prior estimates from observations (Corbosiero et al. 2006). The HIWRAP observations presented in this paper allow a more comprehensive and higher resolution characterization of VRWs and their interaction with the mean vortex than prior observational and most modeling studies.
The details of these new findings are found in the following sections.

\section{b. Airborne radar measurements}

The GH aircraft sampled Matthew for a $\sim 10$-h period between $\sim 1000$ and 2000 UTC 7 October with several overpasses of the storm center and the banded features identified as VRWs. Figure 6 highlights an overpass of the VRWs (shown in the WSR-88D reflectivity scan) and storm center from HIWRAP measurements collected from 1306 to 1345 UTC. The wind vectors at 2-km height are shown to define the swath and the features sampled by HIWRAP. The VRWs are propagating radially and azimuthally (see Fig. 5) through the HIWRAP swath and their reflectivity signature can be seen clearly in the HIWRAP Ku-band data at $2-\mathrm{km}$ height in Fig. 7a. The corresponding stormrelative radial winds (Fig. 7b), vertical winds (Fig. 7c) and vertical vorticity (Fig. 7d) show several perturbations that are collocated or nearly collocated with each other and the reflectivity field (see black arrows in Fig. 7).

The vorticity field (Fig. 7d) shows alternating signed, small-scale vorticity anomalies beyond $100-\mathrm{km}$ radius, which is similar to the outer rainband structure in prior measurements (Didlake and Houze 2011) and simulations (Qiu et al. 2010). Radially inward of the $\sim 100-\mathrm{km}$ radius, the vorticity field is dominated by positive values with banding observed at the $\sim 65-$ and $100-\mathrm{km}$ radii. These vorticity perturbations are not exactly collocated with the reflectivity bands, which were identified with VRWs in section 4a. The small swath width and high resolution of HIWRAP data can be challenging for interpreting smooth, coherent, vorticity bands and not every reflectivity band can be identified with a vorticity band. It is also possible that not every single band is a VRW or that small sections of the largerscale band depart from VRW structure. Nevertheless, the analysis presented in section 4 a clearly identifies the majority of the observed bands as VRWs. (A closer look at the correlation between reflectivity and vorticity bands for this overpass are shown in Figs. 14a and 14b.)

Figure 8 shows Ku-band nadir vertical cross sections of several variables for the HIWRAP overpass in Fig. 7. The reflectivity (Fig. 8a) shows the remnants of the inner eyewall at $20-\mathrm{km}$ radius and the active, main eyewall highlighted by deep convection at $\sim 45-\mathrm{km}$ radius. These features can also be seen clearly in the WSR-88D time series shown in Fig. 5b. For all practical purposes, the current eyewall cycle (labeled in Fig. 2) is complete at this point in time and the focus of this paper is understanding the formation of a new secondary eyewall 


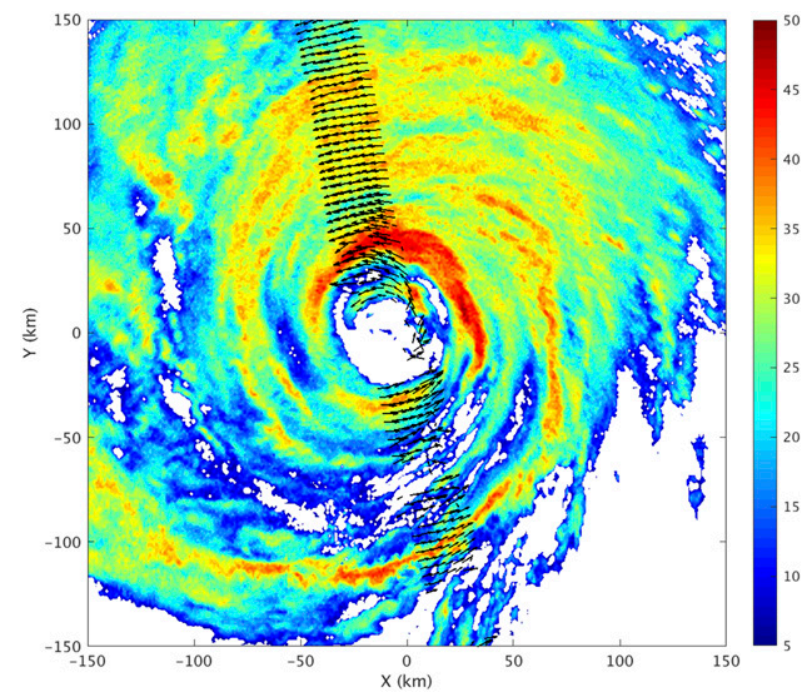

FIG. 6. HIWRAP sampling of Hurricane Matthew (2016) between 1306 and 1345 UTC 7 Oct at 2-km height showing wind vectors overlaid on the closest WSR-88D reflectivity ( $\mathrm{dBZ}$ ) scan to the GH storm-center crossing time (1326 UTC).

(also labeled in Fig. 2) located radially outward from the main eyewall.

Radially outside the main eyewall, thick bands of reflectivity that define the VRWs are discernable (centered at $\sim 80,105$, and $120 \mathrm{~km}$ ), albeit not as clearly as those shown in Fig. 7. The velocity fields were used to calculate the horizontal kinetic energy spectrum in the band region (not shown) revealing peaks in energy from $\sim 12$ - to $15-\mathrm{km}$ wavelength, which represents the radial scales of the VRWs.

The corresponding storm-relative radial winds and vertical winds in Figs. $8 \mathrm{~b}$ and $8 \mathrm{c}$, respectively, show positive perturbations to the background flow at 60-, 80-, $105-$, and $120-\mathrm{km}$ radii (see dashed ovals) that are largely in-phase with each other and tilted radially outward with height. The collocated perturbations in radial and vertical winds extend from the lowest level of HIWRAP data (1-km height) up to midlevels ( $\sim 5-\mathrm{km}$ height). In addition, the radial outflow associated with the VRWs leads to local reductions in the large-scale radial inflow that can be seen most clearly at $\sim 105$ - and $\sim 125-\mathrm{km}$ radii. As mentioned previously, the azimuthal mean radial flow at $\sim 3-\mathrm{km}$ height in the radial band where the VRWs are active was found to be $\sim-3.5 \mathrm{~m} \mathrm{~s}^{-1}$.

The storm-relative tangential winds in Fig. $8 \mathrm{~d}$ show a broad region of $45-50 \mathrm{~m} \mathrm{~s}^{-1}$ winds centered in the main eyewall at $\sim 50-\mathrm{km}$ radius from $\sim 1$ - to $5-\mathrm{km}$ height and a secondary peak at $\sim 75-\mathrm{km}$ radius over the same layer. Perturbations in tangential winds inside the VRWs are not as obvious as those in the radial and vertical wind speed plots. However, perturbations in tangential winds are apparent at high altitudes $(\sim 7-12 \mathrm{~km})$ inside the deep convection at $\sim 55-\mathrm{km}$ radius.

Finally, the vertical cross section of vorticity in Fig. 8e shows large values in the main eyewall, especially at upper levels in the deep convection where peaks
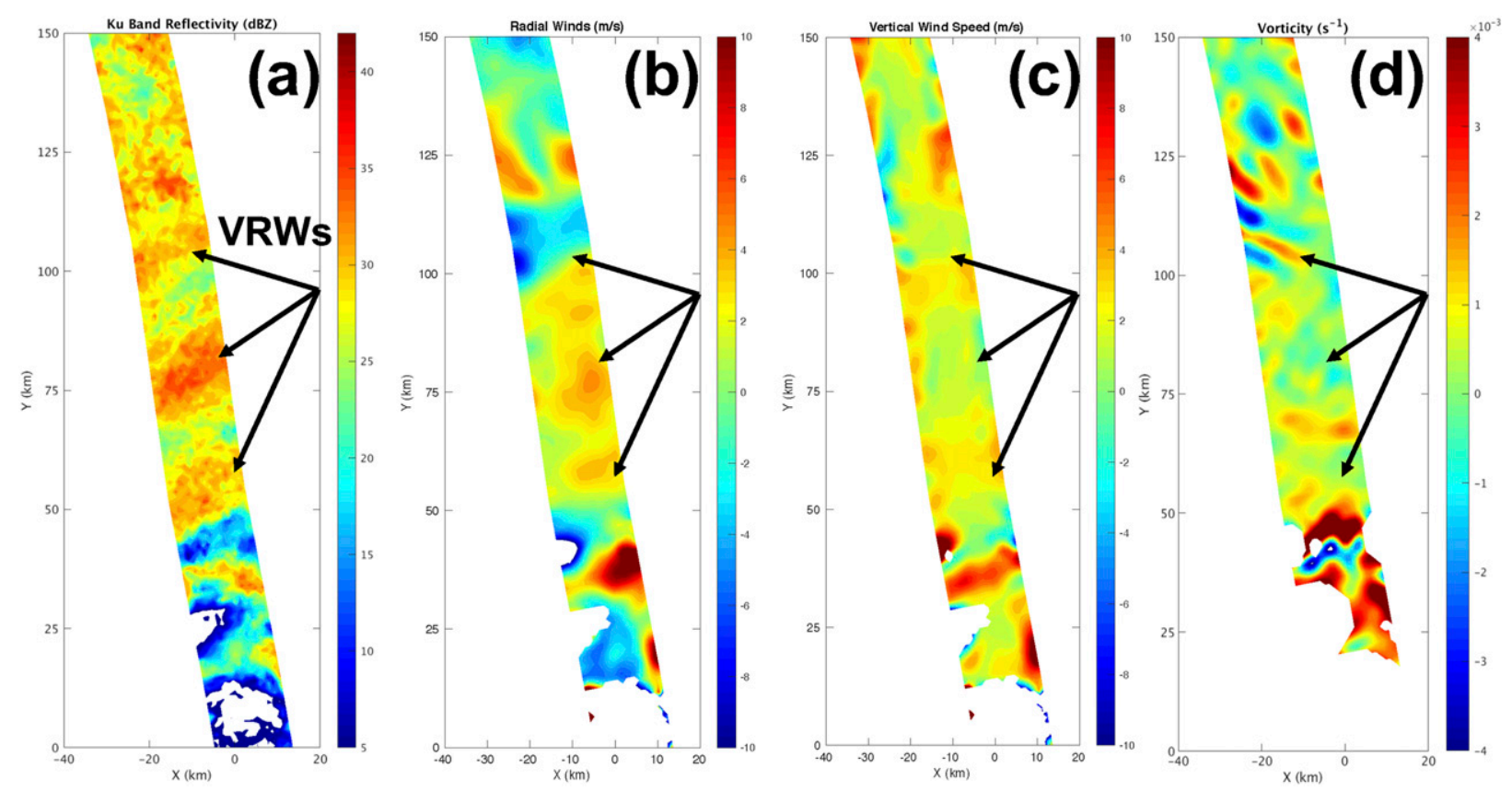

FIG. 7. HIWRAP observations of Hurricane Matthew (2016) between 1306 and 1345 UTC 7 Oct at 2-km height: (a) Ku-band reflectivity (dBZ), (b) storm-relative radial winds $\left(\mathrm{m} \mathrm{s}^{-1}\right)$, (c) vertical winds $\left(\mathrm{m} \mathrm{s}^{-1}\right)$, and (d) relative vertical vorticity $\left(\mathrm{s}^{-1}\right)$. 

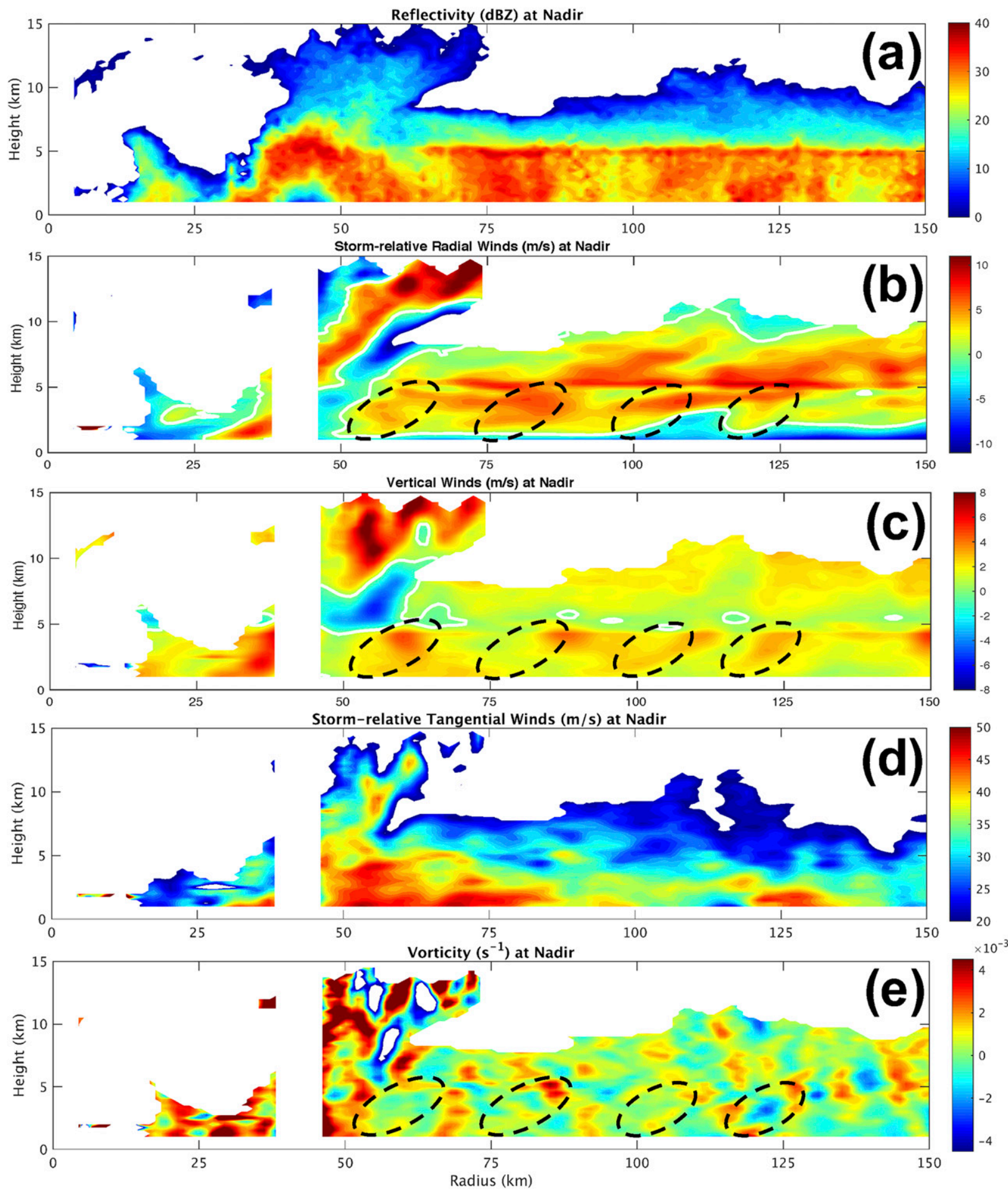

FIG. 8. HIWRAP Ku-band nadir vertical cross sections for the overpass shown in Fig. 7: (a) reflectivity (dBZ), (b) storm-relative radial winds $\left(\mathrm{m} \mathrm{s}^{-1}\right)$, (c) vertical winds $\left(\mathrm{m} \mathrm{s}^{-1}\right)$, (d) storm-relative tangential winds $\left(\mathrm{m} \mathrm{s}^{-1}\right)$, and (e) vertical vorticity $\left(\mathrm{s}^{-1}\right)$. The white curves in (b) and (c) show the zero contour. The dashed black ovals highlight features discussed in the text. 


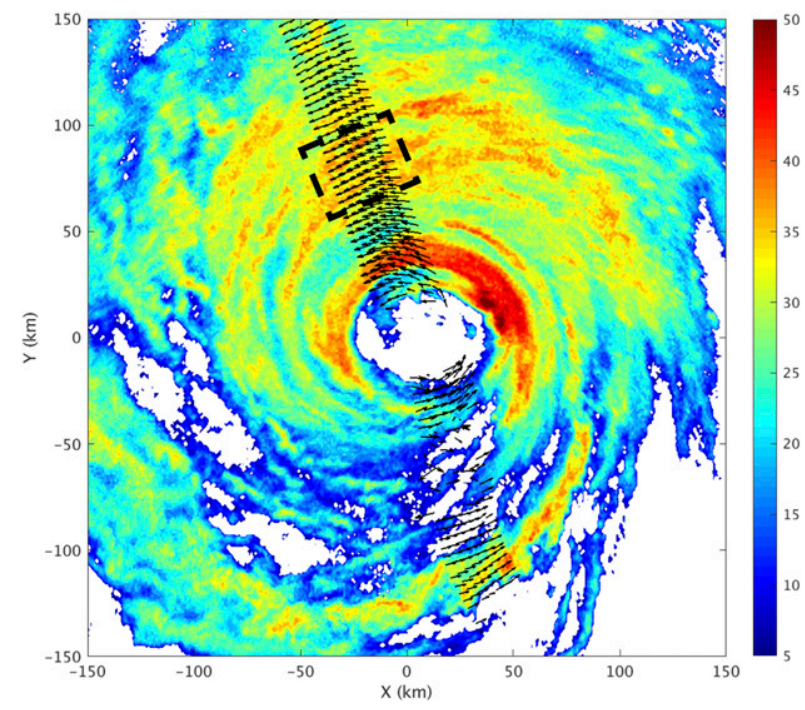

FIG. 9. HIWRAP sampling of Hurricane Matthew (2016) between 1345 and 1424 UTC 7 Oct at 2-km height showing wind vectors overlaid on the closest WSR-88D reflectivity (dBZ) scan to the GH storm-center crossing time (1407 UTC). The dashed black box highlights the $75-100-\mathrm{km}$ radial band where VRWs are active.

of $\sim \pm 7 \times 10^{-3} \mathrm{~s}^{-1}$ are found. The perturbations identified in the previous fields are not obviously collocated with vertically coherent vorticity features, although some positive vorticity signatures are found within the dashed ovals in Fig. 8e. Vertically coherent vorticity features are found just outside the dashed ovals in Fig. 8e, most notably at $\sim 65$ - and $\sim 90$-km radii.

Approximately $20 \mathrm{~min}$ later, the $\mathrm{GH}$ crossed over the VRWs again in a very similar section of the storm between 1345 and 1424 UTC. Figure 9 shows the WSR$88 \mathrm{D}$ reflectivity scan closest to the HIWRAP overpass of the storm center at $\sim 1400$ UTC along with the HIWRAP derived wind vectors at 2-km height. Many individual VRW bands can be identified in the WSR88D data, but in the region of the HIWRAP swath these bands are merging together into a larger reflectivity signature at this time (most notably in the $75-100-\mathrm{km}$ radial range).

Figure 10a shows the storm-relative radial winds in the HIWRAP swath at 2-km height, which reveals a broad region of outflow with largest values of $5-10 \mathrm{~m} \mathrm{~s}^{-1}$ in the $75-100-\mathrm{km}$ radial range (see arrows in Fig. 10a). This broad region of outflow is likely the result of merging VRWs, with each band carrying an individual outflow signature (see Figs. $7 \mathrm{~b}$ and $8 \mathrm{~b}$ from the previous overpass) as they propagate outward against the mean inflow of the vortex. The individual VRW bands are often coupled to the vertical velocity field as shown in Fig. 10b with generally positive covariances (radial outflow coupled to upward vertical motion; see Figs. 14c and $14 \mathrm{~d}$ for an example). Figure 10c also shows regions of elevated storm-relative tangential winds around the 75-km-radius region (see arrow), which may be due to the net VRW activity. Calculations of the angular momentum changes in these regions are analyzed in section 5 to examine this idea more closely. The vorticity field in Fig. 10d shows some positive perturbations in the vicinity of the VRW features at $\sim 75-\mathrm{km}$ radius, but overall is not very informative for this overpass. Again, it is possible that small sections of the larger-scale band depart from VRW structure or that small-scale perturbations in the HIWRAP data are obscuring the larger VRW signal.

Vertical cross sections at nadir for this pass (Fig. 11) show that deep convection in the main eyewall at $\sim 45$ $\mathrm{km}$ radius (Fig. 11a) has decayed relative to the previous overpass and the inner eyewall has almost completely merged with the main eyewall. The broad region of outflow observed in Fig. 10a is evident in Fig. 11b most notably between $\sim 75$ - and $100-\mathrm{km}$ radius extending through a deep layer $(\sim 2-7 \mathrm{~km})$, but with the greatest values in the $\sim 2-5-\mathrm{km}$ layer. Below $\sim 2 \mathrm{~km}$, inflow is present, which extends to higher altitudes at larger radii $(\sim 125-150 \mathrm{~km})$. Two regions of radial outflow perturbations associated with the VRWs are identified in Fig. $11 \mathrm{~b}$ and they are collocated with positive vertical velocity perturbations in the $\sim 2-5$-km layer (Fig. 11c). The positive covariances in radial and vertical winds are also connected to elevated tangential winds in the 75$100-\mathrm{km}$ radial band identified in Fig. 11d and shown in Fig. 10c. The secondary peak in tangential winds is focused at $75-80-\mathrm{km}$ radius in the $\sim 1-3-\mathrm{km}$ layer, which was also found in the previous overpass (Fig. 8d), albeit in a deeper layer.

The merging of the VRW bands and connection to changes in the tangential winds in the $\sim 75-100-\mathrm{km}$ radial zone (focused on 75-80-km region) described above suggests the VRWs may play an important role in the intensity and structure change of this region. Angular momentum budget calculations are performed in the next section that will explore ideas related to the outward-propagating VRWs (observed scales $\sim 12$ $15 \mathrm{~km})$ and their impacts on the larger, vortex scales $(\sim 15-75 \mathrm{~km})$ flow evolution in these regions.

\section{Angular momentum and scale interaction calculations}

a. Methodology

Dynamic budget calculations with measurements are challenging due partly to the limited information provided in space and time for only a few select geophysical 

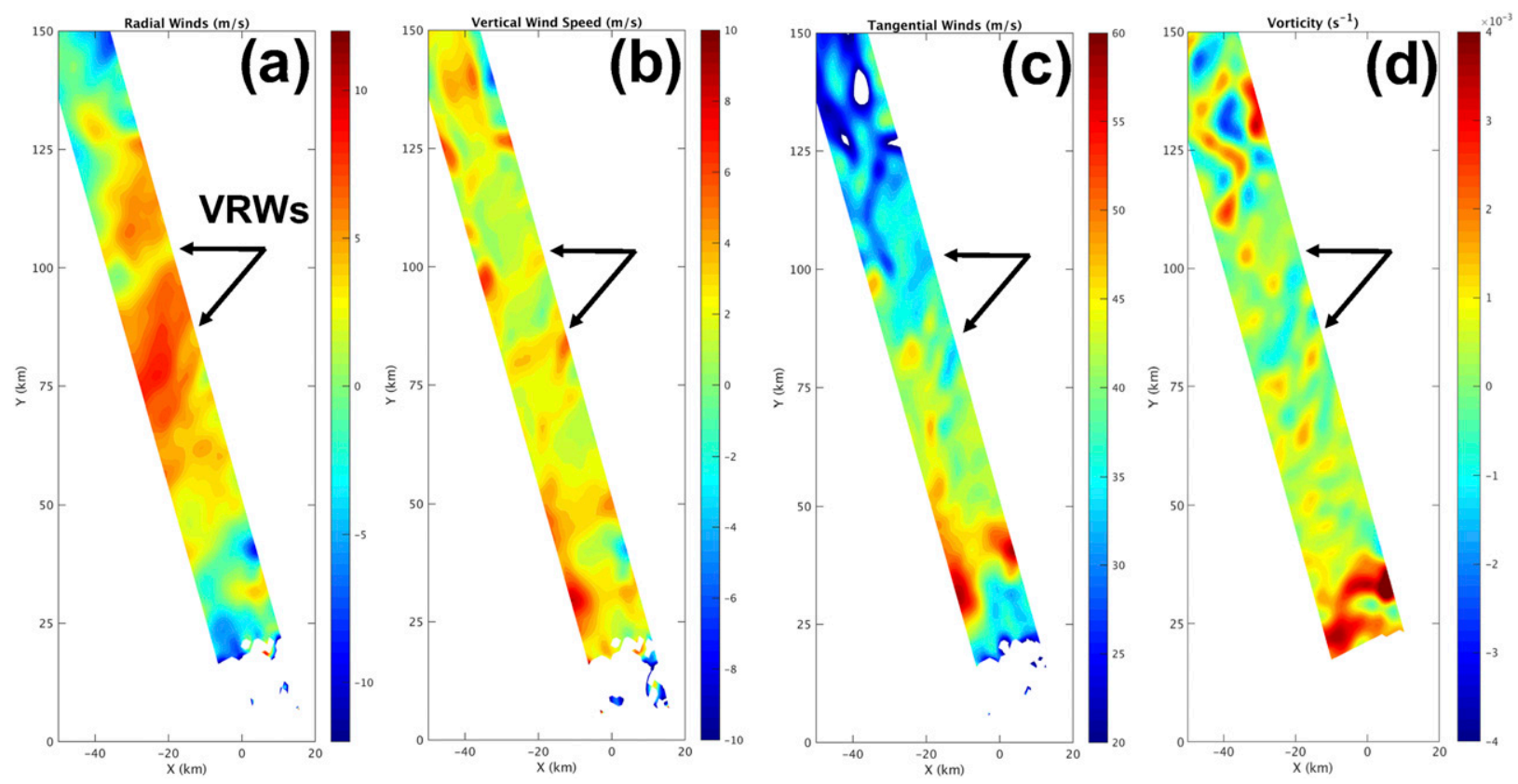

FIG. 10. HIWRAP observations of Hurricane Matthew (2016) between 1345 and 1424 UTC 7 Oct at 2-km height: (a) storm-relative radial winds $\left(\mathrm{m} \mathrm{s}^{-1}\right)$, (b) vertical winds $\left(\mathrm{m} \mathrm{s}^{-1}\right),(\mathrm{c})$ storm-relative tangential winds $\left(\mathrm{m} \mathrm{s}^{-1}\right)$, and (d) relative vertical vorticity $\left(\mathrm{s}^{-1}\right)$.

variables. For HIWRAP, the swath width at the ocean surface from the GH altitude is $\sim 20$ and $\sim 30 \mathrm{~km}$ for the inner $\left(30^{\circ}\right)$ and outer $\left(40^{\circ}\right)$ beams, respectively. While this coverage allows the mapping of localized features and an estimation of the azimuthal mean structure (provided the flight tracks and precipitation are distributed roughly evenly in azimuth), the quantification of azimuthal eddy processes around the vortex is difficult.

To circumvent the azimuthal coverage issue and still provide quantification on the role of the VRWs in the intensity/structure change of the storm, a unique angular momentum budget methodology utilizing azimuthal sectors is employed. Figure 12 shows a typical HIWRAP swath for a northerly flight track through the storm center. An azimuthal sector of the full hurricane can be constructed within the HIWRAP swath with an arc length that matches the swath width at a particular focal radius. The HIWRAP swath data are first interpolated to a storm-centered polar grid $(r, \theta, z)$. Then, an azimuthal sector with a typical focal radius of $\sim 130 \mathrm{~km}$, which is just beyond where the VRWs appear to stagnate, is cut out of the swath. The sector arc lengths are limited to the swath width at $4-5-\mathrm{km}$ height $(\sim 16 \mathrm{~km}$ for the inner beam) due to the HIWRAP geometry and the focus on the low to middle levels of the storm where the VRWs are most prominent. These sectors provide adequate coverage of the VRW dynamics within the HIWRAP swath and sensitivity tests with the inner and outer beams produced similar results.
The flight tracks and HIWRAP geometry provide the most information in the radial dimension, which measures the significant cross-band variability in the VRWs. To understand the roles of different spatial scales in the VRW dynamics occurring in the HIWRAP swath, a scale separation methodology is employed. The three-dimensional flow velocities in the HIWRAP sectors are separated into large-scale and small-scale components by the filtering operation

$$
\tilde{\phi}(r, \theta, z)=\int \phi\left(r^{*}, \theta, z\right) G\left(r-r^{*}\right) d r^{*}
$$

and

$$
\phi^{\prime}(r, \theta, z)=\phi(r, \theta, z)-\tilde{\phi}(r, \theta, z)
$$

where the filter function $G$ and integral operate on the radial dimension and the tilde and prime in (1) and (2) denote the large-scale and small-scale fields, respectively. In this work, a simple top-hat filter is employed (e.g., Leonard 1975) with a window of $15 \mathrm{~km}$, which represents the upper bound for the VRW radial wavelengths detected in the WSR-88D and HIWRAP data $(\sim 12-15 \mathrm{~km})$. The filter was applied in the forward and backward directions to enable zero phase shift. Note that a Fourier cutoff filter was also tried and similar results to the top-hat filter were obtained. 

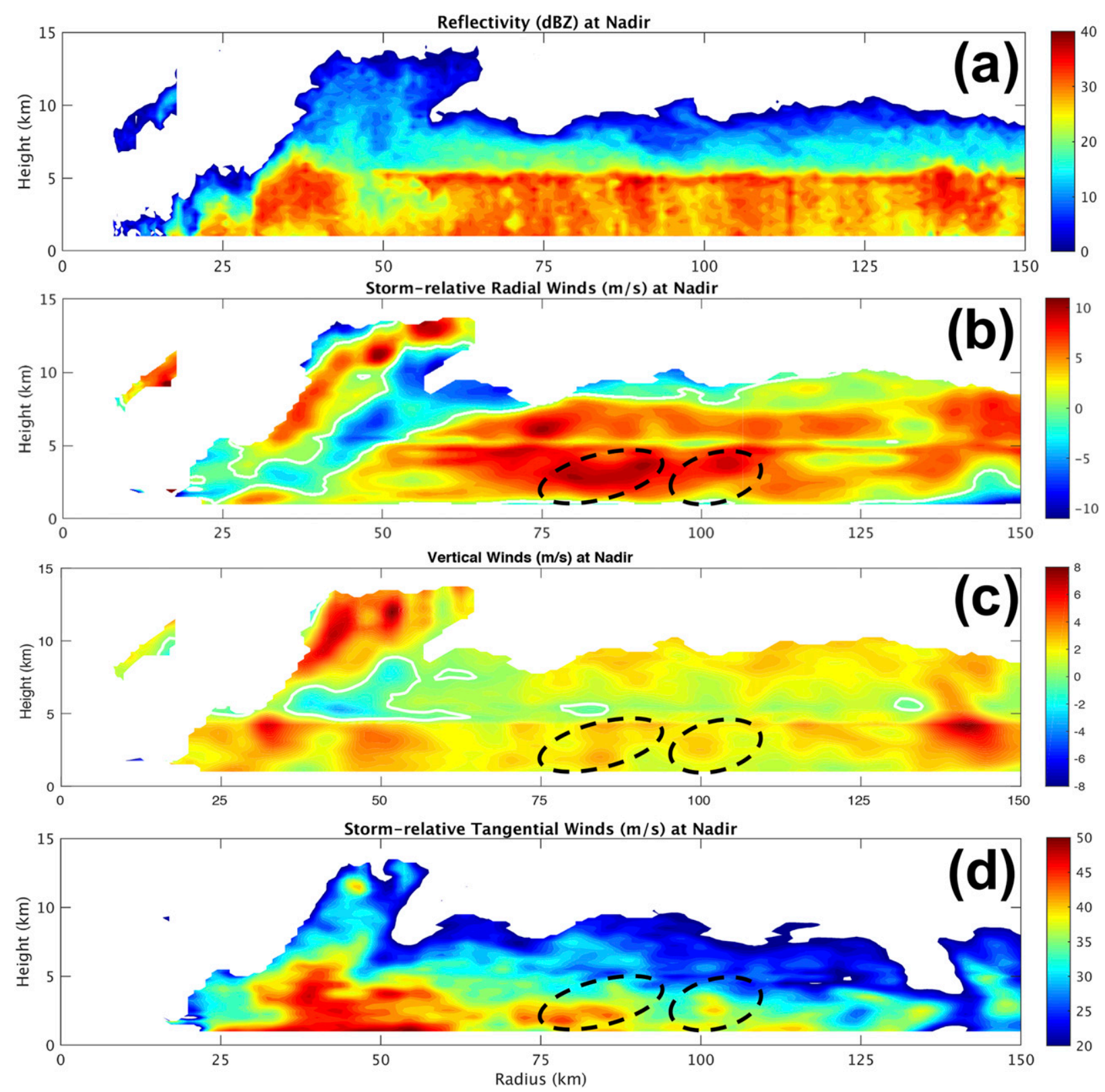

FIG. 11. HIWRAP Ku-band nadir vertical cross sections for the overpass shown in Fig. 10: (a) reflectivity (dBZ), (b) storm-relative radial winds $\left(\mathrm{m} \mathrm{s}^{-1}\right)$, (c) vertical winds $\left(\mathrm{m} \mathrm{s}^{-1}\right)$, and (d) storm-relative tangential winds $\left(\mathrm{m} \mathrm{s}^{-1}\right)$. The white curves in (b) and (c) show the zero contour.

Making the anelastic and $f$-plane approximations and applying (1) to the absolute angular momentum (AAM) equation yields

$$
\begin{aligned}
\frac{\partial \widetilde{M_{a}}}{\partial t} & +\frac{1}{r} \frac{\partial r \tilde{u} \widetilde{M_{a}}}{\partial r}+\frac{1}{r} \frac{\partial \tilde{v} \widetilde{M_{a}}}{\partial \theta}+\frac{1}{\rho_{o}} \frac{\partial \rho_{o} \tilde{w} \widetilde{M_{a}}}{\partial z}=-f_{o} r \widetilde{u_{e}} \\
& -\frac{1}{\rho_{o}} \frac{\partial \tilde{p}}{\partial \theta}-r \mathrm{SFS}+r \mathrm{SGS},
\end{aligned}
$$

where $u, v$, and $w$ are the radial, tangential and vertical velocities, respectively, $f_{o}$ is the Coriolis parameter at the storm center $\left(30^{\circ} \mathrm{N} ; 7.292 \times 10^{-5} \mathrm{~s}^{-1}\right), p$ is the pressure, $\rho_{o}=\rho_{o}(z)$ is a background density profile, and $\widetilde{M}_{a}=r \tilde{v}+f_{o}\left(r^{2} / 2\right)$, is the large-scale AAM. Note that all velocities, except for $u_{e}$ (Earth-relative radial wind) are defined as storm relative by subtracting out the mean storm motion vector from the HIWRAP velocities using reconnaissance aircraft center fixes. The symbols SFS and SGS denote the subfilter-scale and subgrid-scale terms, respectively, which will be described below.

The sector azimuthal mean (refer to Fig. 12 for the geometry) defined as 


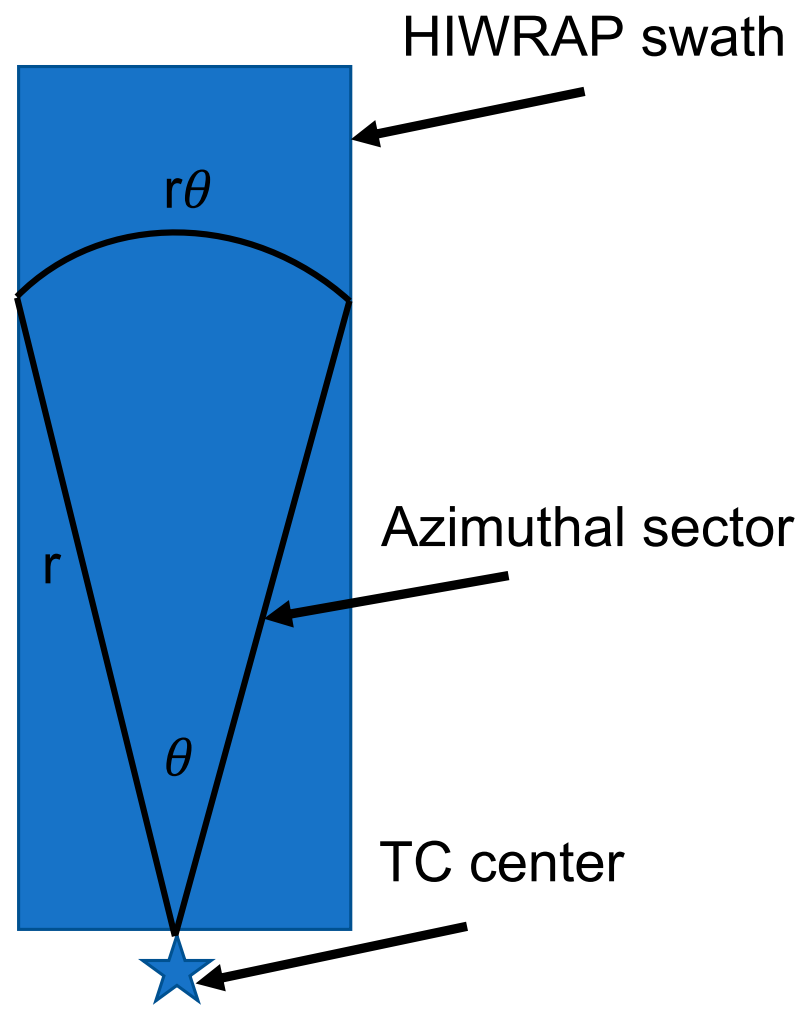

FIG. 12. Schematic that demonstrates the azimuthal sector constructed within the HIWRAP swath used for the angular momentum budget calculations. Note the HIWRAP swath width is $\sim 20-30 \mathrm{~km}$ wide at $1-\mathrm{km}$ height.

$$
\bar{\phi}(r, z)=\frac{1}{r \theta} \int_{0}^{r \theta} \phi(r, \theta, z) r d \theta^{*}
$$

is applied to (3) resulting in the sector azimuthal mean, large-scale AAM tendency equation

$$
\frac{\partial \overline{\bar{M}_{a}}}{\partial t} \cong-\frac{1}{r} \frac{\partial r \overline{\tilde{u}} \overline{\widetilde{M_{a}}}}{\partial r}-\frac{1}{\rho_{o}} \frac{\partial \rho_{o} \overline{\tilde{w}} \overline{\bar{M}_{a}}}{\partial z}-r \overline{\mathrm{SFS}}+r \overline{\mathrm{SGS}}
$$

Note that (5) is written with an approximate equal sign because the sector azimuthal eddy terms were found to be small and they can be neglected for this analysis. In addition, the radial advection of Earth angular momentum was also determined to be small compared to other terms, even at outer radii $(\sim 100 \mathrm{~km})$. The sector azimuthal mean subfilter-scale term $(\overline{\mathrm{SFS}})$ in $(5)$ can be expressed as

$$
\overline{\mathrm{SFS}}=\frac{1}{r^{2}} \frac{\partial r^{2} \overline{\tau_{r \theta}}}{\partial r}+\frac{1}{\rho_{o}} \frac{\partial \rho_{o} \overline{\tau_{z \theta}}}{\partial z},
$$

where

$$
\tau_{r \theta}=(\widetilde{\tilde{u} \tilde{v}}-\tilde{\tilde{u}} \widetilde{\tilde{v}})+\left(\widetilde{\tilde{u} v^{\prime}}+\widetilde{u^{\prime} \tilde{v}}-\tilde{\tilde{u}} \tilde{v^{\prime}}-\tilde{\tilde{v}} \tilde{u^{\prime}}\right)+\left(\widetilde{u^{\prime} v^{\prime}}-\tilde{u^{\prime} v^{\prime}}\right)
$$

and

$$
\tau_{z \theta}=(\widetilde{\tilde{w} \tilde{v}}-\tilde{\tilde{w}} \tilde{\tilde{v}})+\left(\widetilde{\tilde{w} v^{\prime}}+\widetilde{w^{\prime} \tilde{v}}-\tilde{\tilde{w}} \tilde{v^{\prime}}-\tilde{\tilde{v}} \tilde{w^{\prime}}\right)+\left(\widetilde{w^{\prime} v^{\prime}}-\tilde{w^{\prime}} \tilde{v^{\prime}}\right)
$$

Using a first-order closure scheme (e.g., Stull 1988) expressed in polar coordinates for the AAM (azimuthal unit vector), the sector azimuthal mean subgrid-scale term $(\overline{\mathrm{SGS}})$ in $(5)$ is

$$
\overline{\mathrm{SGS}}=\frac{1}{r^{2}} \frac{\partial r^{2}\left(\overline{K_{r}} \frac{\partial \overline{\tilde{v}}}{\partial r}\right)}{\partial r}+\frac{1}{\rho_{o}} \frac{\partial \rho_{o}\left(\overline{K_{z}} \frac{\partial \overline{\tilde{v}}}{\partial z}\right)}{\partial z},
$$

where the eddy viscosities are parameterized following Smagorinsky (1963) taking into account the coordinate system and notations described above,

$$
\overline{K_{r}}=\left(C_{s} \Delta\right)^{2} \sqrt{2\left(\frac{1}{2} \frac{\partial \overline{\tilde{v}}}{\partial r}\right)^{2}}
$$

and

$$
\overline{K_{z}}=\left(C_{s} \Delta\right)^{2} \sqrt{2\left(\frac{1}{2} \frac{\partial \tilde{\tilde{v}}}{\partial z}\right)^{2}}
$$

with $C_{s}$ set to 0.20 and $\Delta$ is 1000 and $250 \mathrm{~m}$ in the radial and vertical dimensions, respectively.

The AAM budget equation [(5)] will be used in the remainder of the paper to interpret the storm dynamics. The term on the lhs is the time tendency of the largescale $(>15 \mathrm{~km})$, sector azimuthal mean AAM. The first and second terms on the rhs of (5) are the large-scale, sector azimuthal mean radial and vertical flux convergence of AAM, respectively. The third term represents the radial and vertical flux convergence of sector mean AAM from small scales, defined as scales between 2 and $15 \mathrm{~km}$. The lower end of this range is the minimum resolvable wavelength on the HIWRAP grid. The inherent energy associated with the VRWs are located within the upper range of these scales, given their wavelength of $\sim 12-15 \mathrm{~km}$. More discussion on this topic will be provided later in the paper.

The sensitivity of the AAM budget results to the chosen scale separation $(15 \mathrm{~km})$ was examined by considering filter windows of 30 and $5 \mathrm{~km}$. These tests revealed that the large-scale contribution, especially the vertical term, is robust while the small-scale terms can change some due to the incorporation of more and less energy for the 30- and 5-km scales, respectively. The 15$\mathrm{km}$ scale is used in the remainder of the paper because of the connection to the VRWs and does not affect the core results and conclusions. 
The SFS components involve the divergence of stress tensors defined in (7) and (8), where the terms on the rhs are the Leonard, cross, and Reynolds stresses or fluxes. These fluxes are defined as in Germano (1986), which also appear in large eddy simulation and measurement studies (e.g., Lilly 1967; Leonard 1975; Sullivan et al. 2003). Each flux term represents a specific nonlinear interaction among scales with terms subtracted off to account for variability in the filtering operations across general grids. The Germano (1986) definition allows for each flux term to be uniquely interpreted, regardless of the filter function employed.

The Leonard flux represents nonlinear interactions between the large-scale components of the flow. The cross flux represents the nonlinear interaction of large-scale and small-scale components of the flow such as the impacts of VRWs on the vortex scales. The Reynolds flux represents nonlinear interactions between small-scale components of the flow and their projection onto the large scales. Finally, the fourth term on the rhs in (5) is an estimate of the impact of dynamics not resolved on the HIWRAP grid (scales $<2 \mathrm{~km}$ ) on the large-scale flow. This term was found to be quite small in the weakly turbulent outer regions of the storm examined here and is not discussed in detail.

\section{b. Budget analysis for the 1306-1345 UTC period}

Figure 13 illustrates the large-scale, sector azimuthal mean AAM, tangential, radial, and vertical winds for the 1306-1345 UTC overpass focusing on the outercore region below the melting level. The AAM field (Fig. 13a) shows increasing values with radius and generally decreasing values with height, which is common in hurricanes. The AAM field increases with height between 1 and $1.5 \mathrm{~km}$ at most radii with larger increases up to $\sim 3.0-\mathrm{km}$ height in the $\sim 100-115-\mathrm{km}$ radial band. These increases in AAM with height are due to strong tangential winds protruding out to large radii from $\sim 1.5$ to $3.0-\mathrm{km}$ height (Fig. 13b). The tangential wind protrusions are located in significant radial outflow (peak of $\sim 6 \mathrm{~m} \mathrm{~s}^{-1}$; Fig. 13c) with inflow, likely from the mean vortex, below $1.5-\mathrm{km}$ height. The inflow gets stronger and deeper with radius (peak values of $\sim-8 \mathrm{~m} \mathrm{~s}^{-1}$ at $125-\mathrm{km}$ radius) especially in the $100-115-\mathrm{km}$ radial band. At the leading edge of this radial band $(\sim 100 \mathrm{~km})$, the outflow and inflow collide producing upward motion (Fig. 13d) that extends from the lowest levels up to 4-km height where the largest values of $3 \mathrm{~m} \mathrm{~s}^{-1}$ are found. Note that downward motion is not found in Fig. 13d because the total values are weak and these fields have been filtered and averaged over the sector.
Figure 14 shows the small-scale (scales $\leq 15 \mathrm{~km}$ ), sector azimuthal mean fields for the same overpass. The vorticity field (Fig. 14a) reveals vertically coherent, upright anomalies with radial wavelengths of $\sim 12$ $15 \mathrm{~km}$ and generally larger amplitudes at farther radii. The phase lines of the positive vorticity anomalies are drawn in Fig. 14a and they are copied onto the reflectivity field in Fig. 14b. These phase lines show strong correlations between small-scale, positive vorticity and reflectivity bands at $\sim 85-, \sim 95-, \sim 107-$, and $\sim 118-\mathrm{km}$ radii. The positive vorticity band at $\sim 68-\mathrm{km}$ radius (Fig. 14a) is not correlated with a positive reflectivity band and may locally depart from VRW structure. Overall, the correlations described above provide clearer evidence that the majority of reflectivity bands tracked in the WSR-88D and HIWRAP observations are VRWs.

The phase lines of the radial wind perturbations (Fig. 14c) are sloping radially outward with height and show peak values of $2-3 \mathrm{~m} \mathrm{~s}^{-1}$. The phase lines drawn for the radial wind perturbations are copied onto the vertical wind perturbations (peak values of $1-2 \mathrm{~m} \mathrm{~s}^{-1}$; Fig. 14d), which reveals positive momentum fluxes (i.e., $\left.u^{\prime} w^{\prime}>0\right)$ in the VRWs over most of the domain, especially in the $\sim 90-125-\mathrm{km}$ radial band. Positive correlations in the vorticity field with the radial and ver tical winds can also be found in the $\sim 90-125-\mathrm{km}$ radial band.

In summary, the large-scale, sector mean fields in Fig. 13 reveal that strong tangential winds are located at large radii above the low-level inflow. At outer radii ( $\sim 100-\mathrm{km}$ radius), a secondary circulation has developed and together these characteristics suggest the building process of a secondary eyewall, at least within the HIWRAP swath. Considering that the convectively coupled VRWs are the dominant physical features found within the HIWRAP swath and they are associated with positive vertical fluxes of radial momentum, and to some extent vorticity (Fig. 14), it is reasonable to propose that they are playing a meaningful role in the secondary eyewall formation process. Budgets of AAM are presented next to examine this idea more closely and identify the radial locations where the secondary eyewall development is most prominent.

Figure 15 shows the terms that compose the AAM budget equation in (5) for the 1306-1345 UTC overpass. Figure $15 \mathrm{a}$ is the time tendency term, computed as a summation of all terms on the rhs of (5), revealing significant increases in large-scale AAM from $\sim 1.5-\mathrm{km}$ height and above centered at 70-75- and $\sim 100-\mathrm{km}$ radii. At low levels (below 1.5-2-km height) there are decreases in AAM, especially between 110- and $120-\mathrm{km}$ radii. The time tendency of AAM is governed 

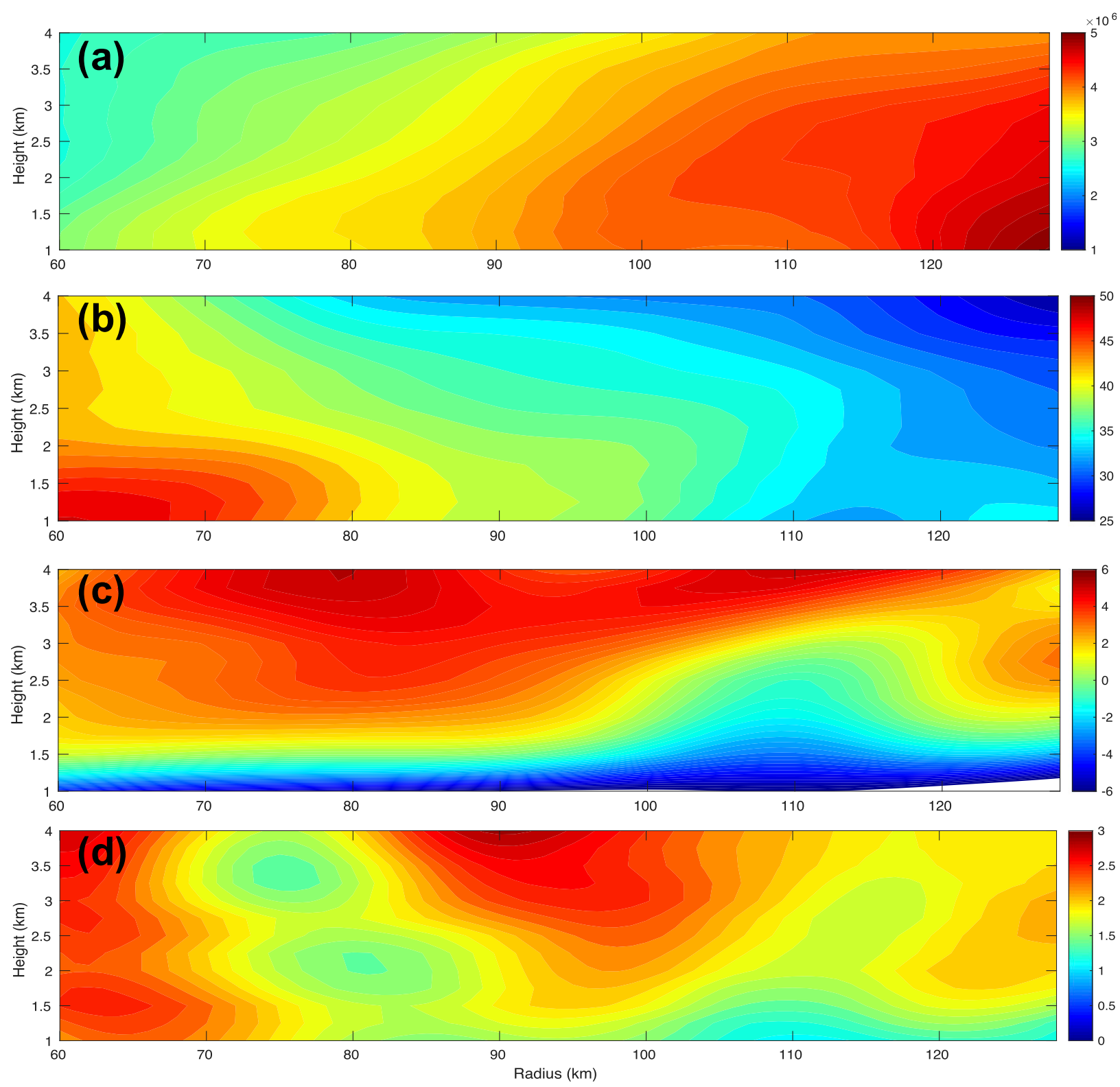

FIG. 13. Large-scale, sector azimuthal mean fields for the 1306-1345 UTC HIWRAP overpass: (a) AAM ( $\left.\mathrm{m}^{2} \mathrm{~s}^{-1}\right)$, (b) tangential wind $\left(\mathrm{m} \mathrm{s}^{-1}\right),(\mathrm{c})$ radial wind $\left(\mathrm{m} \mathrm{s}^{-1}\right)$, and $(\mathrm{d})$ vertical wind $\left(\mathrm{m} \mathrm{s}^{-1}\right)$.

principally by the large-scale vertical flux convergence term (Fig. 15b) and radial flux convergence term (Fig. 15c).

The vertical term (Fig. 15b) dominates the time tendency with centers of spinup located at 70-75-, $100-$, and $120-\mathrm{km}$ radii extending from $\sim 1.5-\mathrm{km}$ height and above. At the lower levels there is spindown, especially at outer radii, which may reflect the overall storm weakening trend discussed in section 3 . The radial term (Fig. 15c) shows an acceleration dipole with spinup centered at $100-\mathrm{km}$ radius and spindown at $120-\mathrm{km}$ radius confined to the $1.5-3.5-\mathrm{km}$ layer.
The domain averaged vertical gradients in AAM are approximately $8-10$ times larger than the radial gradients, which is why the large-scale vertical term dominates the budget. The dominance of the vertical term indicates that baroclinic dynamics are playing a central role in the system evolution as Matthew enters the midlatitude flow with significant environmental vertical wind shear.

The only other terms in (5) that make a significant contribution to the AAM time tendency are the smallscale (SFS) vertical and radial terms, even though they are much smaller than their corresponding large-scale 

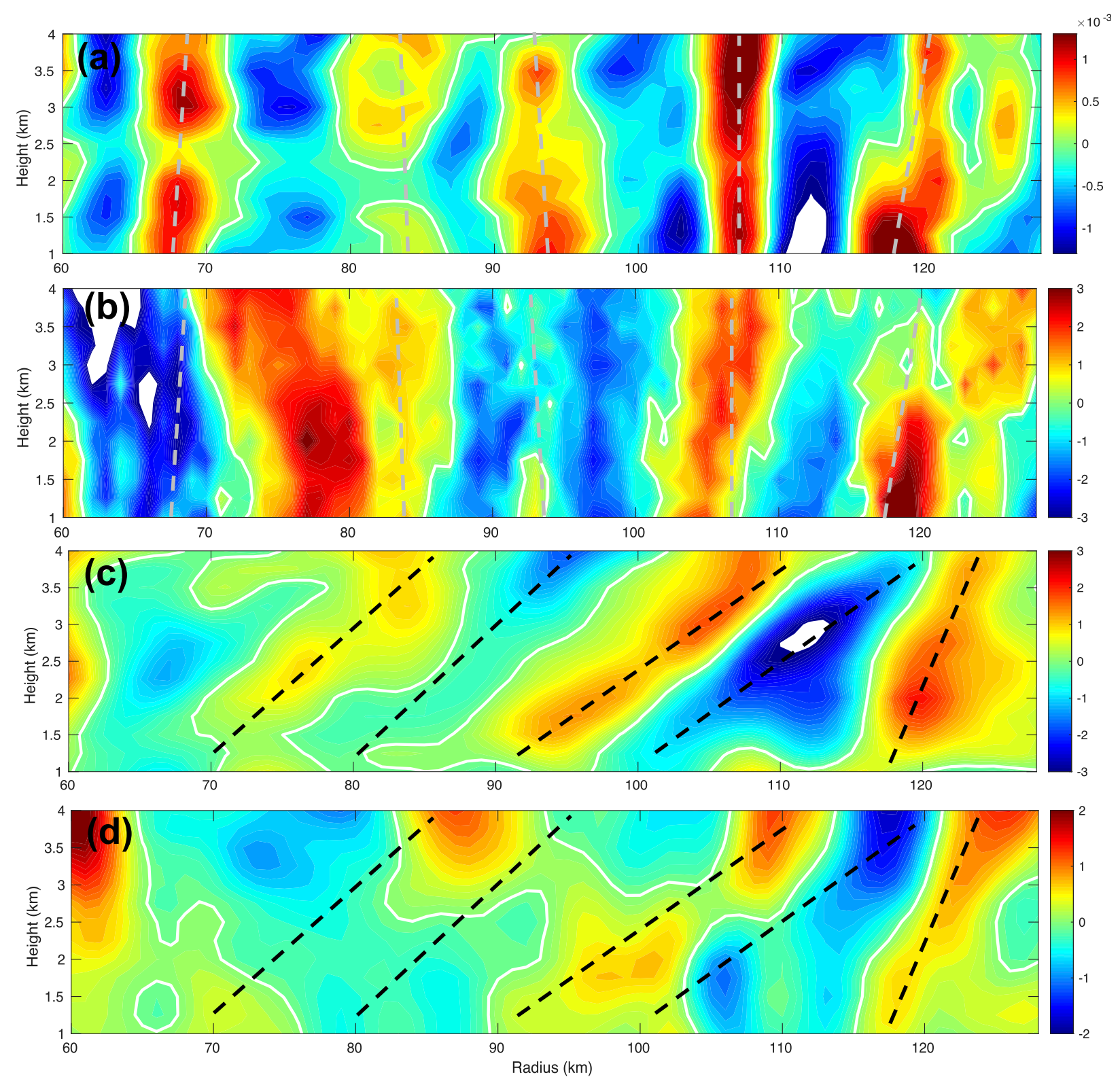

FIG. 14. Small-scale, sector azimuthal mean fields for the 1306-1345 UTC HIWRAP overpass: (a) vorticity (s ${ }^{-1}$ ), (b) reflectivity (dBZ), (c) radial wind $\left(\mathrm{m} \mathrm{s}^{-1}\right)$, and (d) vertical wind $\left(\mathrm{m} \mathrm{s}^{-1}\right)$. The white curves show the zero contour and the dashed lines highlight the phase lines of the perturbations discussed in the text.

terms. The SFS vertical term (Fig. 15d) has the largest impact due to the larger AAM gradients as discussed above. There are two main radial regions where acceleration is noted above $1.5-\mathrm{km}$ height, the $70-80-\mathrm{km}$ band and the $100-\mathrm{km}$ region (denoted by dashed circles in Fig. 15d). Deceleration is noted radially outside the $110-\mathrm{km}$ radius at $1-$ and $3.5-\mathrm{km}$ heights. These positive/negative acceleration regions are similar to those observed in the large-scale vertical and radial terms (Figs. 15b,c), which is consistent with the notion of the small scales impacting the large-scale dynamics through nonlinear interactions. The SFS radial term (Fig. 15e) has a very weak contribution to the overall budget and is only shown for completeness, but the largest values are located at the $110-\mathrm{km}$ radius where the small-scale radial and tangential winds are strong.

Figure 16 shows the decomposition of the net SFS vertical term into the three flux components to gain more insight into the nonlinear dynamics. Focusing 

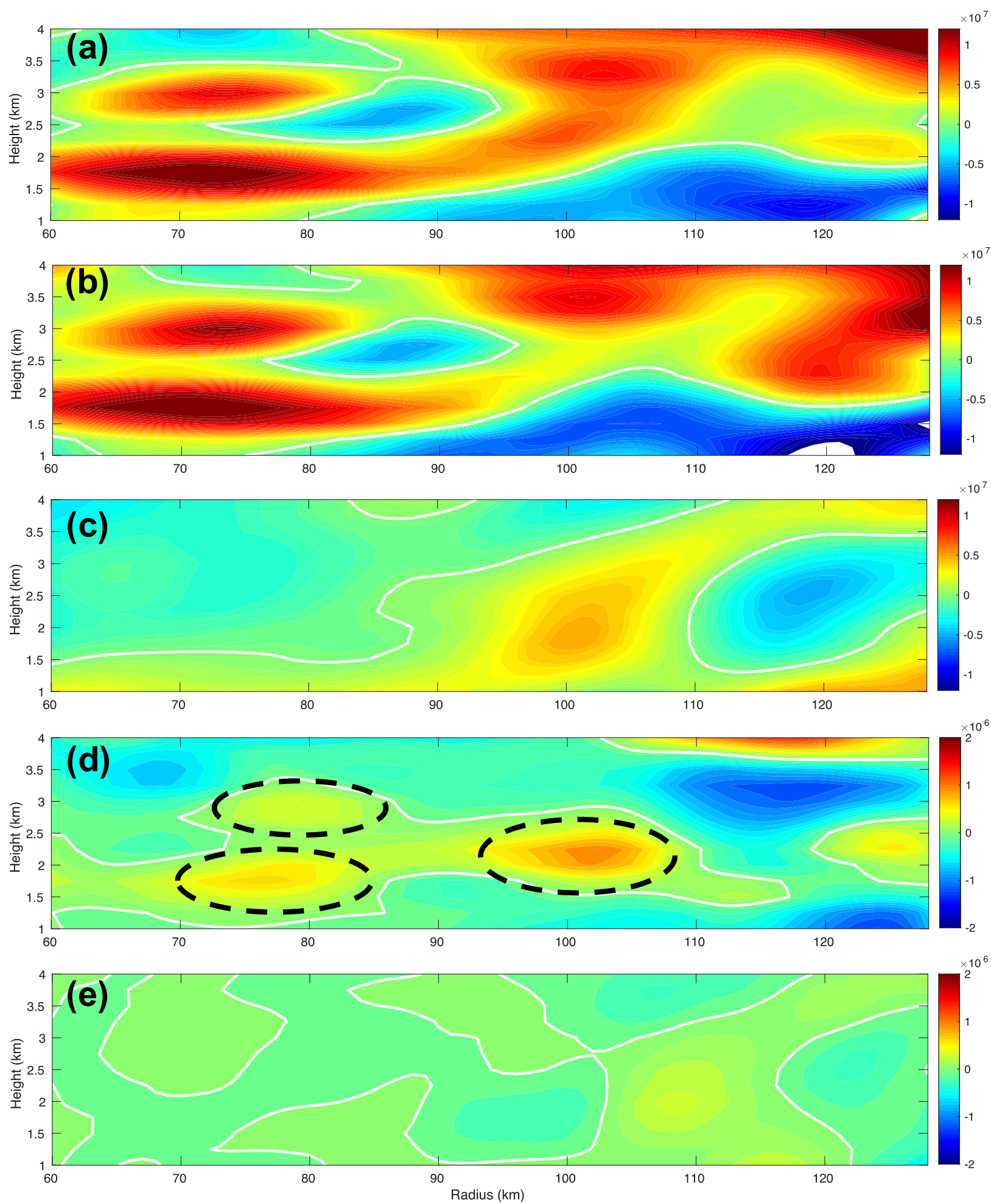

FIG. 15. Terms from the large-scale, sector azimuthal mean AAM budget equation [(5)] for the 1306-1345 UTC HIWRAP overpass: (a) AAM time tendency, (b) large-scale vertical flux convergence of AAM, (c) large-scale radial flux convergence of AAM, (d) net SFS (small scale) vertical flux convergence of AAM, and (e) net SFS (small scale) radial flux convergence of AAM. The units in all panels are $\mathrm{m}^{2} \mathrm{~s}^{-1} \mathrm{~h}^{-1}$. Note the smaller color bar range in (d) and (e). The dashed black circles in (d) highlight regions discussed in the text. 

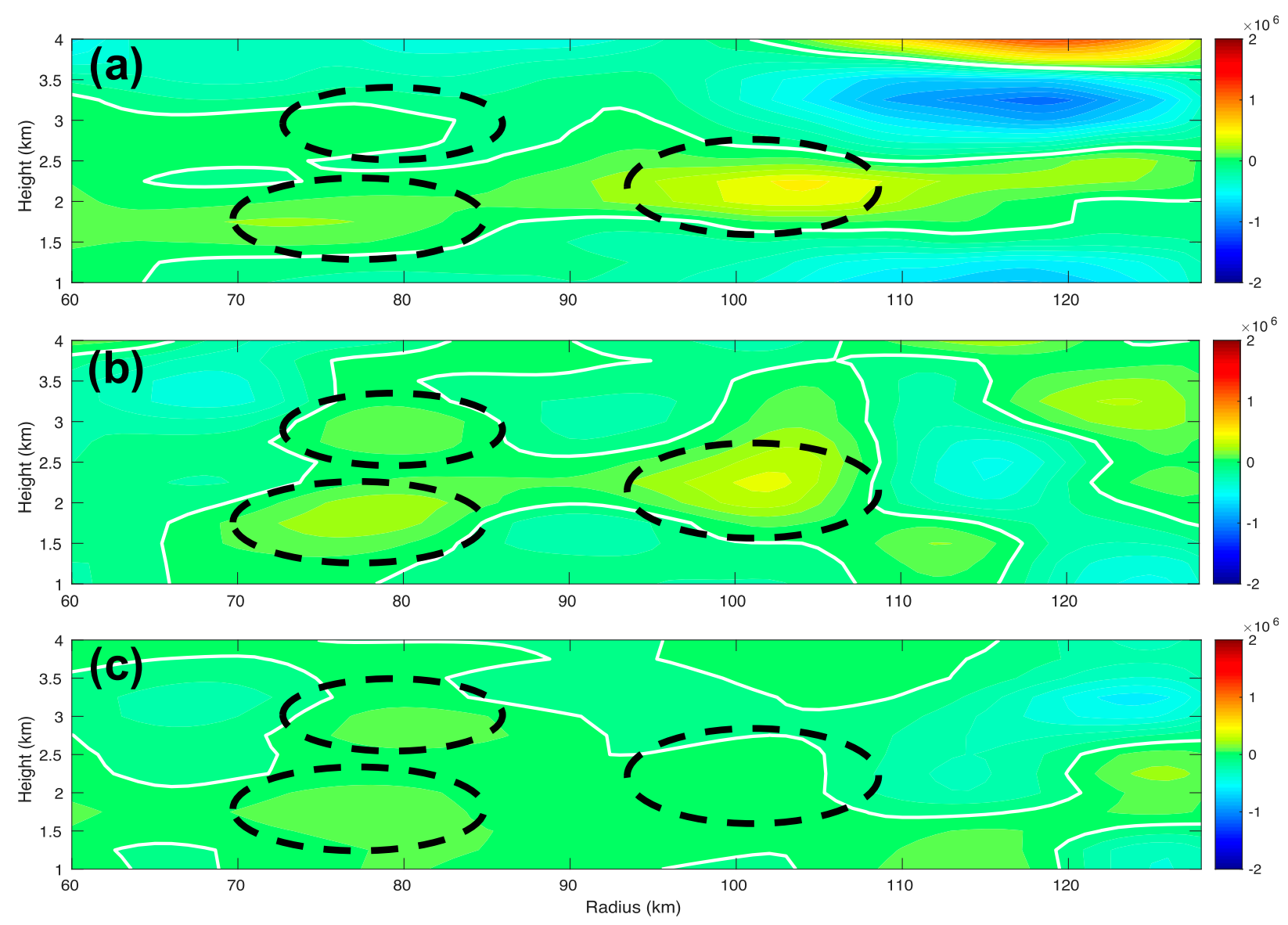

FIG. 16. Terms that compose the net SFS (small scale) vertical component from the AAM budget equation [(5)] for the 1306-1345 UTC HIWRAP overpass: (a) Reynolds stress, (b) cross stress, and (c) Leonard stress. The units in all panels are $\mathrm{m}^{2} \mathrm{~s}^{-1} \mathrm{~h}^{-1}$. The dashed black circles highlight regions discussed in the text.

on the regions highlighted in the net SFS vertical term (dashed circles), the Reynolds flux (Fig. 16a) plays the largest role followed by the cross flux (Fig. 16b) and Leonard flux (Fig. 16c). The Reynolds and cross fluxes are reinforcing each other especially in the $\sim 100-\mathrm{km}$ radius region from 2 - to $3-\mathrm{km}$ height and in the $70-80-\mathrm{km}$ radial band. Physical interpretation of these terms and the full AAM budget equation [(5)] are provided next.

\section{c. Physical interpretation}

The HIWRAP observations are sampling the VRWs and their transport of AAM nearly instantaneously as the aircraft makes a radial transect above the storm. In reality, however, the waves have been evolving and interacting with the flow for a period of time prior to the measurements and their integrated effects are embedded in the data. There are two primary ways that the VRWs will impact the large-scale hurricane vortex: projection onto the low-wavenumber ( 0 and 1$)$ dynamics and nonlinear interactions, which can also include feedback processes.
As the VRWs break from the main eyewall, their cyclonic rotation enables projection onto both the wavenumber-1 and wavenumber-0 fields (e.g., see Fig. 3) with the most significant activity in the $\sim 75-125$ $\mathrm{km}$ radial band. Consequently, the VRW kinematics (radial and vertical fluxes of AAM) will be embedded in the wavenumber- 1 and wavenumber- 0 fields, which enables significant contribution to the large-scale AAM budget terms. The HIWRAP observations and budget analyses portray a representative azimuthal section of the VRWs in this radial region and the bands project very strongly onto the large-scale, sector azimuthal mean fields. The HIWRAP analyses show that the VRWs are convectively coupled in a baroclinic environment and they act to lift and converge larger AAM found at low levels $(<2 \mathrm{~km})$ up to higher levels. This vertical convergence of AAM accelerates the tangential winds and produces a secondary wind maximum in the swath. The VRWs are also transporting higher AAM from the inner core to the outer core where they meet the inflow from the vortex-scale secondary 

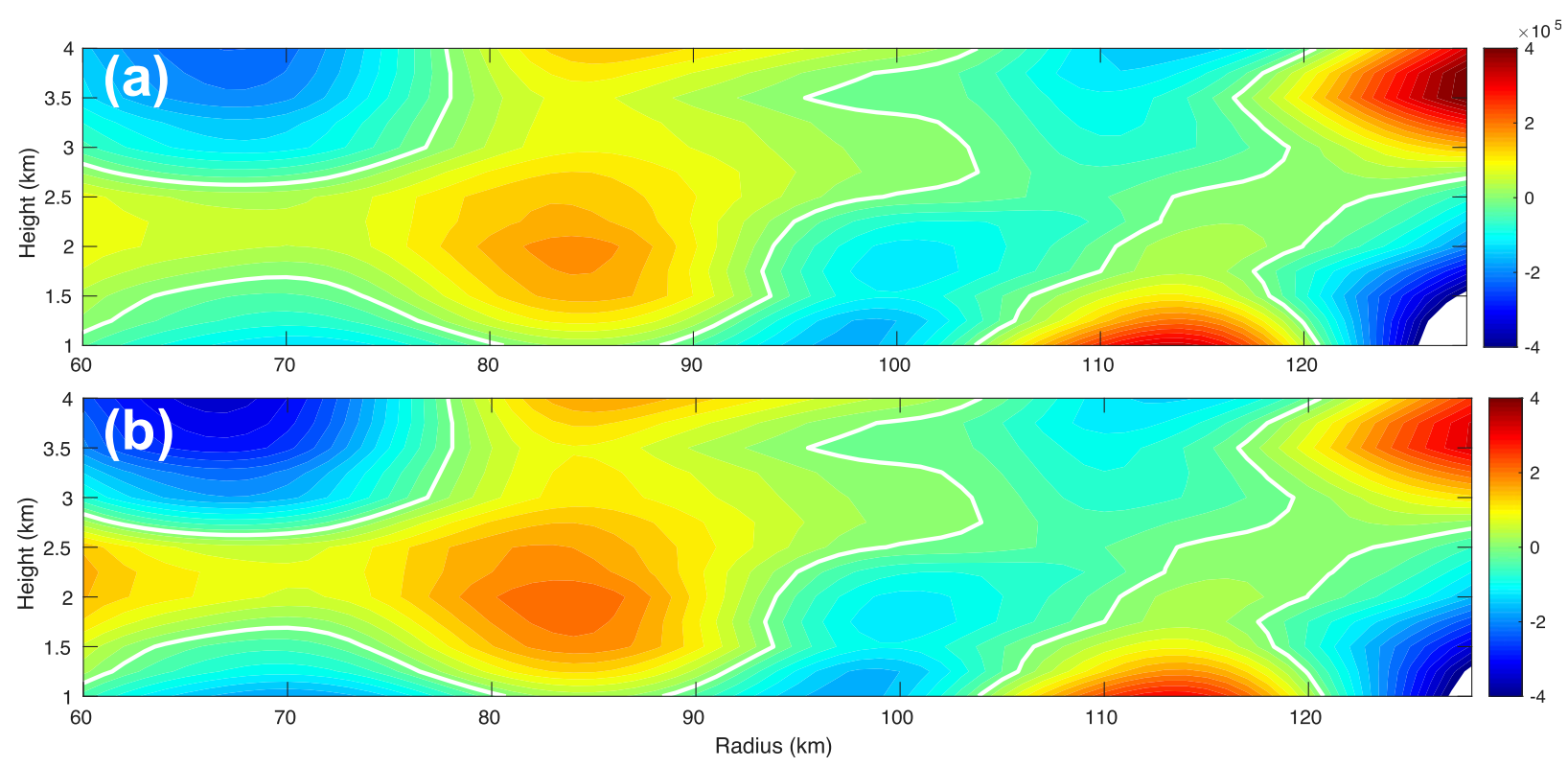

FIG. 17. Change in large-scale, sector azimuthal mean (a) AAM $\left(\mathrm{m}^{2} \mathrm{~s}^{-1}\right)$ and (b) tangential wind $\left(\mathrm{m} \mathrm{s}^{-1}\right)$ between the 1306-1345 and $1345-$ 1424 UTC HIWRAP overpasses.

circulation, resulting in a spinup signature at $\sim 100$ $\mathrm{km}$ radius.

Advective nonlinearities allow interactions between scales to occur (including wave-wave and wave-mean flow), which can leave an imprint on the flow at scales larger than the primary forcing. This means that the VRWs, which were estimated to have radial wavelengths of $\sim 12-15 \mathrm{~km}$, can conceivably produce changes in the flow at larger scales through scale interactions. Feedback processes, such as VRWs interacting with other small-scale features to excite convection is also occurring in the storm, but is not discussed in this paper.

While the large-scale dynamics are playing the dominant role in the AAM evolution, they are connected to the small scales through the nonlinear interactions outlined above. The small scales are where the intrinsic nature of the VRWs are found. The small-scale vertical fluxes of AAM associated with the VRWs are most prominent in similar regions as the large-scale terms, which indicates they are forcing the outer-core spinup through the interaction of individual waves projected onto the large scales (Reynolds flux contribution). The direct interaction of the small scales with the large scales is represented through the cross flux contribution, which is reinforcing the same regions that are accelerating the large-scale AAM. Although the net effect of these nonlinear interactions at any one instant are small, they can build up more significant large-scale effects when integrated over the VRW forcing time period. The total VRW forcing time period may be on the order of $\sim 12 \mathrm{~h}$ based on the WSR$88 \mathrm{D}$ data shown in Fig. 5. Larger nonlinear effects are anticipated inside the boundary layer and in the innercore region.

Additional VRW observations and budget analyses for the 1345-1424 UTC time period (section 5d) show very similar results and physical interpretation to those discussed here for the 1306-1345 UTC period. Given the robust results and the discussion outlined in this section, we assess that the VRWs are playing an important role in the secondary eyewall formation process primarily through their large-scale vertical and radial effects.

Figures $17 \mathrm{a}$ and $17 \mathrm{~b}$ show the change in large-scale, sector azimuthal mean AAM and tangential wind, respectively, between the 1306-1345 and 1345-1424 UTC overpasses. These two overpasses covered similar regions (cf. Figs. 6 and 9) with an azimuthal distance and time offset of $\sim 10-15 \mathrm{~km}$ and $\sim 20 \mathrm{~min}$, respectively. Perfect correlations between the AAM budget predictions and the observed change in AAM/tangential wind with these space and time differentials are not expected, especially for quantitative comparisons. As a result, the focus is more on qualitative comparisons. The time offset is the more important factor due to the fast dynamic time scale of the sector azimuthal mean fields when compared to the full azimuthal mean fields.

Despite these time/space offsets, the general structure is revealing. A substantial increase in AAM 
$\left(\sim 1-2 \times 10^{5} \mathrm{~m}^{2} \mathrm{~s}^{-1}\right)$ and tangential winds $(\sim 1.5-$ $2.5 \mathrm{~m} \mathrm{~s}^{-1}$ ) in the $\sim 75-90-\mathrm{km}$ radial band centered at $\sim 2-\mathrm{km}$ height is evident in Figs. 17a and 17b, respectively. As described previously, this radial band and height location contains significant increases in AAM from the time tendency term (Fig. 15a), which is driven by the large-scale (Fig. 15b) and small-scale (Fig. 15d) vertical fluxes provided by the VRWs. The general structure of the VRWs in this radial band and height location are shown in Figs. 7 and 8. Increases of AAM/tangential wind in other regions of Fig. 17 do not match up as well with the AAM budget calculations probably due to space/time offsets.

The physical interpretation of the AAM budget results discussed above is supported by the traditional, vortex azimuthal mean and eddy partitioning budgets of Qiu et al. (2010). In their idealized simulation, they showed evidence for outward-propagating VRWs and an AAM acceleration signature at outer radii and $\sim 1-$ $2-\mathrm{km}$ height due to azimuthal mean and eddy processes. The authors suggested that the contribution of the VRWs to the azimuthal mean secondary circulation played the larger role in the outer-core spinup and observed increases in mean tangential wind. However, Qiu et al. (2010) ultimately determined that the VRWs are playing an indirect role in the secondary eyewall formation by facilitating the axisymmetrization of convective-scale vorticity anomalies. This is in contrast to the present study, where the VRWs are observed to play a more direct role in the secondary eyewall formation.

\section{d. Budget analysis for the 1345-1424 UTC period}

Figure 18 shows the terms from the AAM budget equation for the 1345-1424 UTC overpass, which occurred in a similar location to the 1306-1345 UTC overpass, but with a time offset of $\sim 20 \mathrm{~min}$. The largescale and small-scale, sector azimuthal mean velocity fields are not shown for this overpass to make the discussion concise, but they can be roughly inferred from the total fields shown in Fig. 11.

The time tendency term (Fig. 18a) has some similar qualitative structure to the prior overpass with two acceleration dipoles centered at $\sim 80$ - and $\sim 100-\mathrm{km}$ radii. These acceleration dipoles are associated with the VRWs (see Fig. 11). The inner acceleration dipole has moved $\sim 10 \mathrm{~km}$ radially outward, but spinup of the large-scale, sector azimuthal mean tangential winds centered at $\sim 2-\mathrm{km}$ height is still predicted in this overpass. Consistent with the prior overpass, the vertical flux convergence term (Fig. 18b) dominates the AAM time tendency with the same acceleration dipoles visible. The radial flux convergence term (Fig. 18c) shows a wide spindown region inside of the $\sim 85$-km radius and largely spinup outside. This structure is due to a significant region of large-scale outflow centered at $80-85-\mathrm{km}$ radius (Fig. 11b), which is the result of merging VRWs identified in the ground-based (Fig. 9) and airborne radar data (Figs. 10 and 11).

The small-scale (SFS) vertical term (Fig. 18d) shows deceleration of the large-scale flow at low-levels $(\sim 1-2$ $\mathrm{km}$ height) with larger values and a deeper spindown signature as the radius increases. Acceleration is noted above these levels with peak spinup signatures at $\sim 90$ - and $\sim 105-\mathrm{km}$ radii in the $2-4-\mathrm{km}$ layer. This structure is similar to the large-scale vertical term (Fig. 18b), which indicates that the native scales of the VRWs are weakly forcing the large-scale dynamics through nonlinear interactions associated with vertical fluxes of AAM. The small-scale radial term (Fig. 18e) has a minor contribution to the large-scale AAM budget. Figure 19 shows the breakdown of the SFS vertical term into the specific nonlinear interaction components.

The Reynolds flux (Fig. 19a) is the largest contributor to the net SFS vertical term with a broad region of deceleration radially outside the $90-\mathrm{km}$ radius from $\sim 2 \mathrm{~km}$ and below and acceleration above these levels with peak values at $\sim 90$ - and $\sim 105-\mathrm{km}$ radii. The cross flux (Fig. 19b) and Leonard flux (Fig. 19c) are also contributing to the acceleration of these regions, but with progressively smaller values except at far radii.

While some of the AAM budget terms described here have different structure compared to the previous overpass (due to space/time offsets), the physical interpretation and implications for the dynamics of the storm are basically the same. The budget time tendencies in the 1306-1345 and 1345-1424 UTC time periods show significant increases in angular momentum and tangential winds in the $\sim 70-100-\mathrm{km}$ radial band associated with the VRW activity. Does a secondary peak in tangential winds in this region exist from data other than HIWRAP?

Figure 20a shows the flight tracks of three Air Force radial legs through Matthew at $\sim 3$-km height between 1209 and 1542 UTC 7 October overlaid on the WSR$88 \mathrm{D}$ reflectivity scan at 1325 UTC. The flight tracks cover a wide range of azimuths on the northern side of the storm and sample the VRWs and outer-core region studied here. Figure 20b shows the radial profiles of tangential winds for these three flight legs revealing clear secondary peaks in tangential winds clustered in the $75-80-\mathrm{km}$ radial band, which further indicates the development of a wavenumber-one secondary eyewall. Note that the large peak at $\sim 85-\mathrm{km}$ radius is near an 

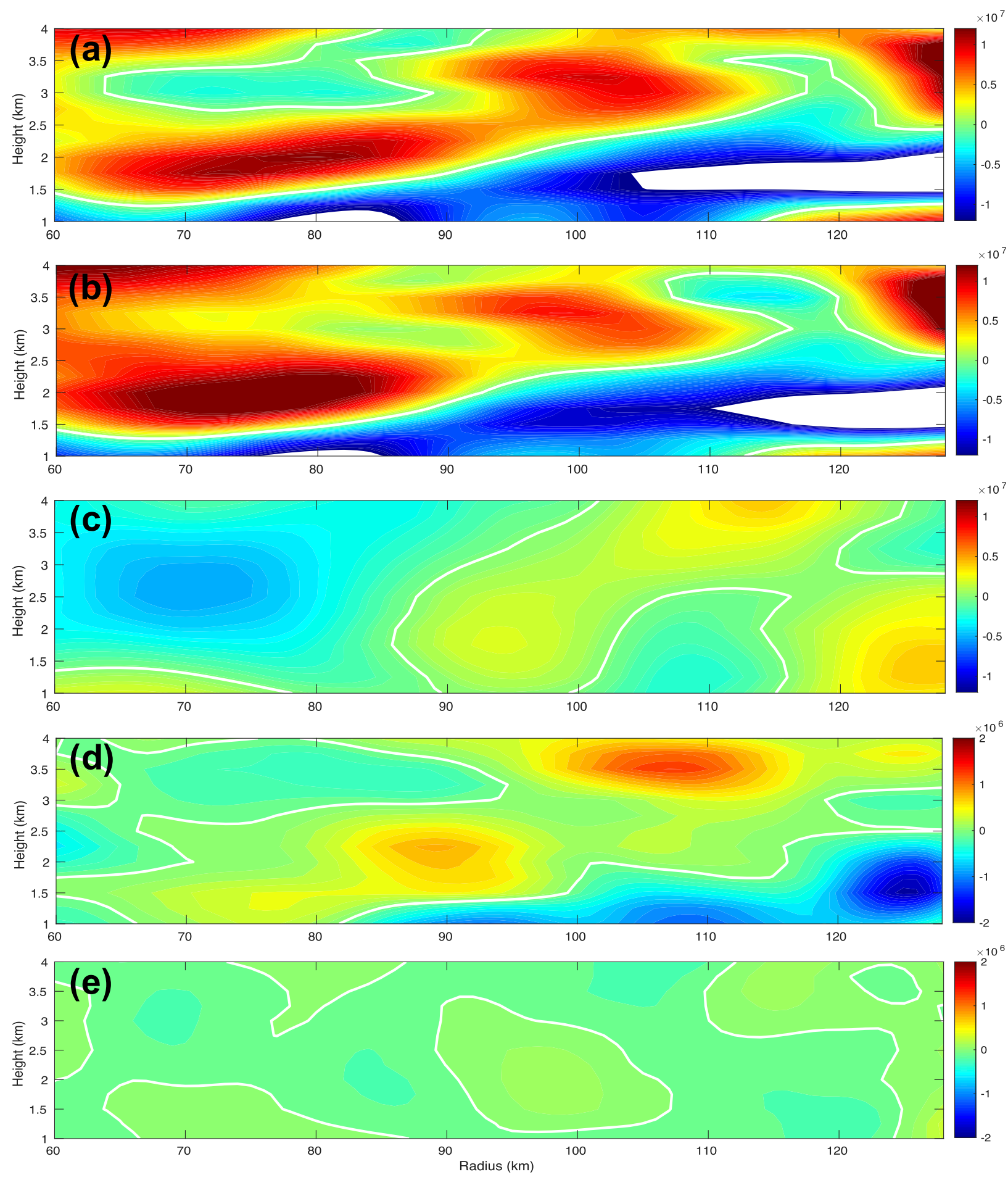

FIG. 18. As in Fig. 15, but for the 1345-1424 UTC HIWRAP overpass only.

aircraft turn and might be due to the departure from a radial leg.

There are limitations to the HIWRAP observations. While the HIWRAP analyses cannot characterize the exact radial location of this secondary eyewall, they do show substantial increases in large-scale, sector mean AAM and tangential wind in close proximity. Furthermore, although the HIWRAP swaths are only 

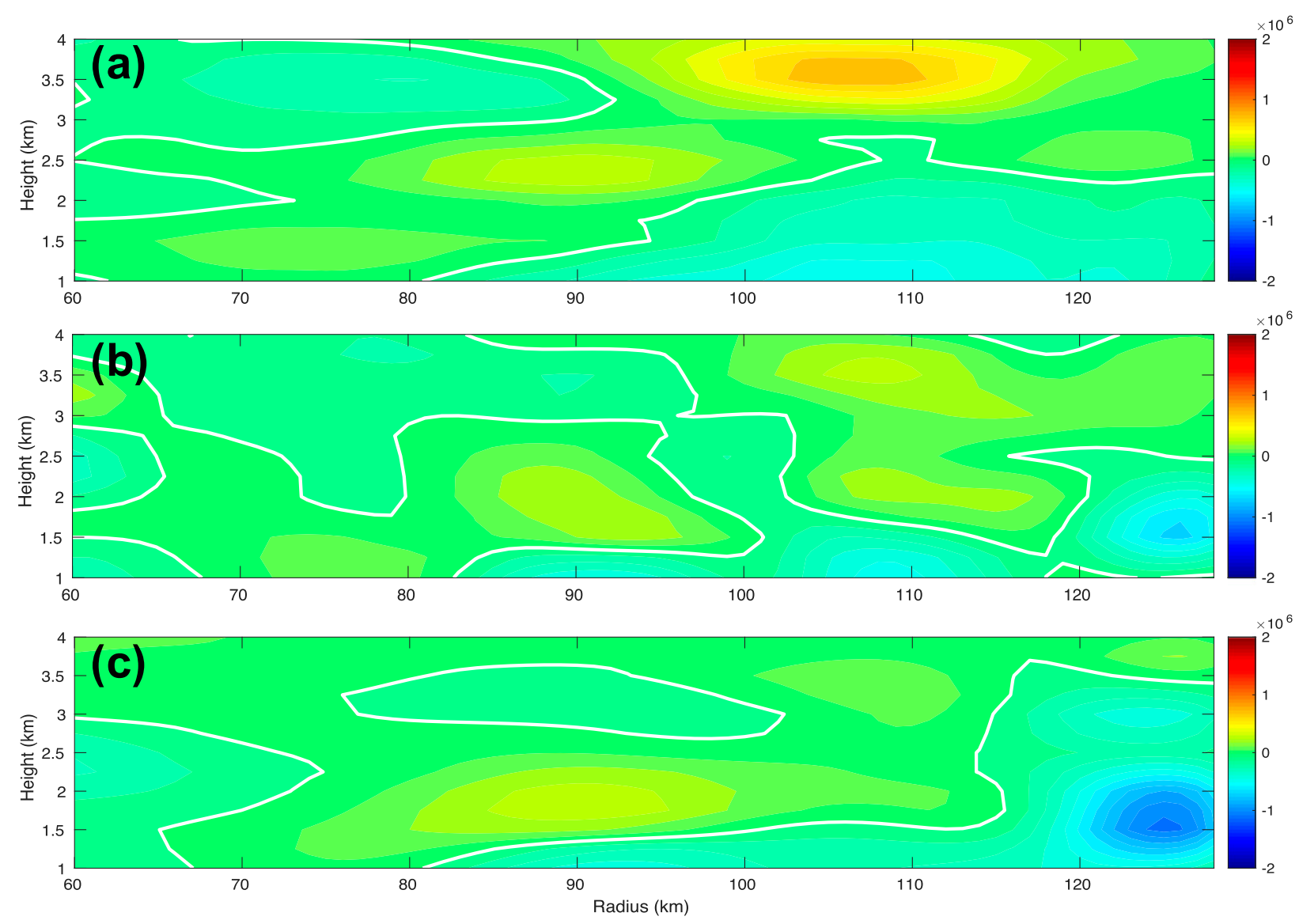

FIG. 19. As in Fig. 16, but for the 1345-1424 UTC HIWRAP overpass only.

characterizing a portion of the VRW bands that are linked to the observed spinup signatures, we expect similar dynamical mechanisms are operating across the full azimuthal extent of the bands on the northern side of the storm. This assumption is consistent with the flight-level data in Fig. 20, which shows secondary tangential wind maxima at very similar radial locations distributed in azimuth.
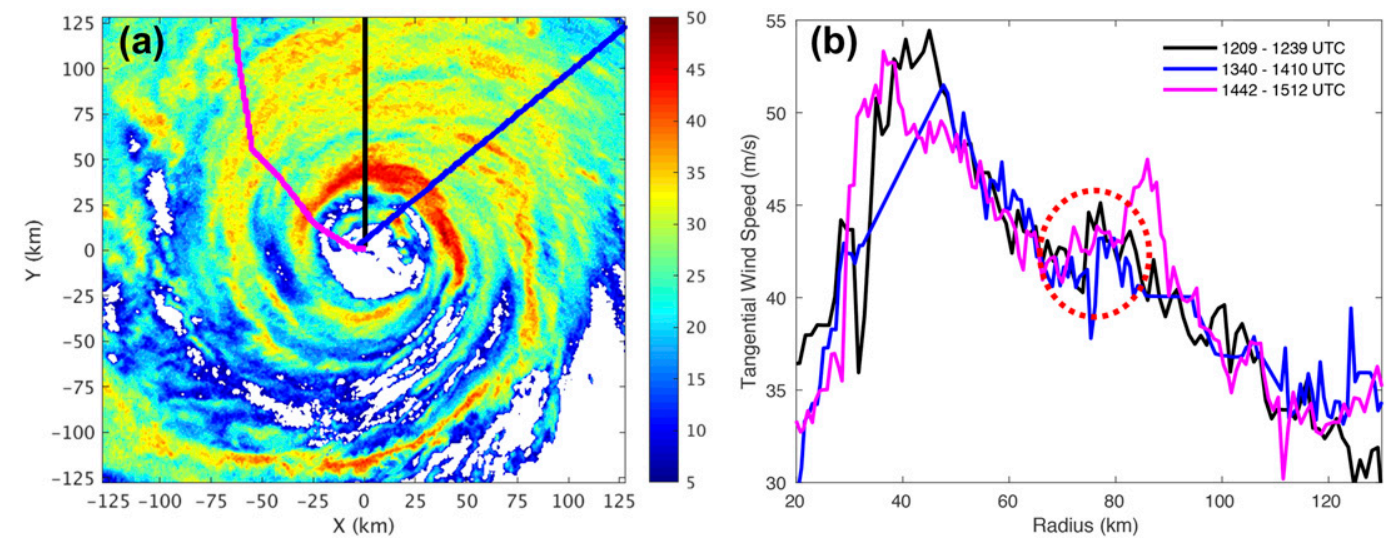

FIG. 20. Air Force flight-level data at $\sim 3-\mathrm{km}$ height on 7 Oct 2016 showing (a) the location of three radial legs overlaid on the 1325 UTC WSR-88D reflectivity (dBZ) scan and (b) radial profiles of tangential winds for the tracks shown in (a). The line colors for the time ranges in (a) and (b) are the same. Secondary peaks in tangential winds are highlighted by the dotted red circle. 


\section{Summary and conclusions}

In this paper, the structure and evolution of Hurricane Matthew (2016) during a slow weakening trend and eyewall replacement cycle is examined from a suite of observations taken during the NOAA Sensing Hazards with Operational Unmanned Technology (SHOUT) field experiment. Ground-based radar data from a NOAA WSR-88D in conjunction with airborne radar measurements from the NASA HIWRAP instrument on the Global Hawk aircraft provided a relatively high-spatial-resolution $(\sim 1 \mathrm{~km})$ and hightemporal-resolution ( $\sim 5 \mathrm{~min})$ dataset for studying the details of the system evolution.

Ground-based radar measurements between $\sim 1000$ and 2000 UTC 7 October 2016 revealed small-scale ( $\sim 12-\mathrm{km}$ radial wavelength) reflectivity bands breaking off the main eyewall in the downshear-right quadrant of the storm and spreading in radius and azimuth where their wavelength increased to $\sim 15 \mathrm{~km}$. The groundbased data were used to estimate the propagation characteristics of the bands to identify their intrinsic nature. The intrinsic, measured azimuthal phase speeds of the bands were $\sim-14.5 \mathrm{~m} \mathrm{~s}^{-1}$ at $50-\mathrm{km}$ radius and the outward radial speeds (for the wavenumber- 2 component) were $\sim 8.50 \mathrm{~m} \mathrm{~s}^{-1}$.

The speed calculations indicate that the spiral bands propagated against the cyclonic tangential flow and radial inflow of the azimuthal mean vortex. The intrinsic azimuthal phase speeds of the bands are consistent with calculations based on barotropic vortex Rossby wave (VRW) theory (MK97). These results, together with the detected collocation or near-collocation of reflectivity and vorticity bands indicates that the features analyzed in this paper are trailing spiral, sheared VRWs.

The HIWRAP observations revealed the detailed structure of the convectively coupled VRWs, which were most active in the $\sim 75-125-\mathrm{km}$ radial band. The individual spiral bands had correlations with positive perturbation vorticity and carried positive covariances in radial and vertical velocity and often tangential winds. In one overpass, the VRW bands were merging together to create a strong, wide swath of outflow from $\sim 2$ - to 5 $\mathrm{km}$ height or higher with peak total values $>10 \mathrm{~m} \mathrm{~s}^{-1}$. The peak perturbation radial, vertical and tangential winds in the HIWRAP data associated with the VRWs were $\sim \pm 3, \pm 2$, and $\pm 4 \mathrm{~m} \mathrm{~s}^{-1}$, respectively. At levels below 2-km height, inflow associated with the azimuthal mean vortex was found with total values of $\sim-5 \mathrm{~m} \mathrm{~s}^{-1}$.

Flight-level and HIWRAP winds showed the development of tangential wind maxima in the outer core of the storm and the HIWRAP data were used to understand the dynamics of this secondary eyewall formation process. A new angular momentum budget methodology utilizing azimuthal sectors was presented that allows an understanding of the storm physics with narrowswath radar measurements. Filtering of the governing equations into large-scale ( $>15-\mathrm{km}$ radial wavelength) and small-scale $(\leq 15 \mathrm{~km})$ components enables explicit insight into the nonlinear dynamics of scale interactions and the role of the VRWs in the storm structure change.

The results showed that the large-scale vertical flux convergence of angular momentum played the largest role in the time tendency term with centers of acceleration in the $\sim 70-80$ - and $\sim 100-\mathrm{km}$ radial regions from $\sim 2$ - to $4-\mathrm{km}$ height. The secondary tangential wind maxima were observed in the $75-80-\mathrm{km}$ radial region in flight-level data at $\sim 3-\mathrm{km}$ height. These regions are connected with the convectively coupled VRWs and they act to lift and converge higher angular momentum found at low-levels (below $\sim 2-\mathrm{km}$ height). Deceleration of the low-level angular momentum was observed, which appears consistent with the overall storm weakening trend.

The VRWs were also transporting high angular momentum from the inner-core region to the outer-core region where they meet the vortex-scale inflow. This leads to an acceleration dipole at outer radii in the largescale radial flux convergence term. In one overpass, a secondary circulation was apparent at $\sim 100-\mathrm{km}$ radius, but this feature did not seem robust in subsequent sampling. The VRW effects are found in the large-scale, sector azimuthal mean terms because of the strong projection of the bands onto the low-wavenumber $(0$ and 1) vortex kinematic fields as well as from the integrated effects of prior dynamic interactions.

Changes in the large-scale angular momentum from nonlinear interactions with the smaller scales contributed to a weak, but nonnegligible forcing from the net vertical flux component. This forcing is primarily driven by the Reynolds stress, which represents the interactions between individual VRWs and their projection onto the large-scale, sector mean fields. The cross flux, which quantifies the direct forcing of the large scales from the small scales, also contributes to the net forcing. The action of the net, small-scale vertical flux term is to reinforce the forcing from the large-scale vertical flux and when integrated over a time window where the VRWs are active ( $~ 12 \mathrm{~h}$ based on WSR-88D data), they can build up more significant effects. The angular momentum budget results described above are generally supported by the idealized modeling study of Qiu et al. (2010). They utilized standard vortex azimuthal mean/eddy decomposition budgets and showed spinup signatures and the development of a secondary eyewall at outer radii from VRWs. The authors suggested that the contribution of the VRWs to the 
azimuthal mean secondary circulation played the larger role in the outer-core spinup.

Overall, the results of the present study indicate that the convectively coupled VRWs are leading to a direct spinup of the outer-core tangential wind field and are associated with the development of a wavenumber-one secondary eyewall. This is in contrast to studies that have described $V R W s$ as playing an indirect role in the secondary eyewall formation by expanding the outer envelope of vorticity, which assists in the axisymmetrization of convective-scale vorticity anomalies. Observations from the HIWRAP and WSR-88D measurements do not show the axisymmetrization of convective-scale vorticity anomalies in the radial region where the secondary eyewall is forming $(\sim 75-80 \mathrm{~km})$. Instead, we have identified coherent VRW bands that are propagating into this radial region, merging with other bands and projecting energy into the larger-scale flow. Future research should analyze the coupling of the boundary layer (not measured by HIWRAP), including symmetric and asymmetric physics, to the VRW mechanism identified in this paper to develop a more comprehensive understanding of the secondary eyewall formation and evolution process.

Acknowledgments. We thank Dr. Lihua Li for his engineering efforts on HIWRAP during SHOUT. This research was supported by the NASA Weather and Atmospheric Dynamics program (Grant NNH16ZDA001N-WEATHER) and the NOAA SHOUT field experiment. We thank three anonymous reviewers for their constructive comments that helped to improve the clarity and quality of this paper.

\section{REFERENCES}

Abarca, S. F., and K. L. Corbosiero, 2011: Secondary eyewall formation in WRF simulations of Hurricanes Rita and Katrina (2005). Geophys. Res. Lett., 38, L07802, https://doi.org/ 10.1029/2011GL047015.

—_ _ - , and D. Vollaro, 2011: The World Wide Lightning Location Network and convective activity in tropical cyclones. Mon. Wea. Rev., 139, 175-191, https://doi.org/ 10.1175/2010MWR3383.1.

Bell, M. M., M. T. Montgomery, and W. C. Lee, 2012: An axisymmetric view of concentric eyewall evolution in Hurricane Rita (2005). J. Atmos. Sci., 69, 2414-2432, https://doi.org/ 10.1175/JAS-D-11-0167.1.

Black, M. L., and H. E. Willoughby, 1992: The concentric eyewall cycle of Hurricane Gilbert. Mon. Wea. Rev., 120, 947-957, https://doi.org/10.1175/1520-0493(1992)120<0947:TCECOH> 2.0.CO;2.

Corbosiero, K. L., J. Molinari, A. R. Aiyyer, and M. L. Black, 2006: The structure and evolution of Hurricane Elena (1985). Part II: Convective asymmetries and evidence for vortex Rossby waves. Mon. Wea. Rev., 134, 3073-3091, https://doi.org/ 10.1175/MWR3250.1.
Didlake, A. C., and R. A. Houze Jr., 2011: Kinematics of the secondary eyewall observed in Hurricane Rita (2005). J. Atmos. Sci., 68, 1620-1636, https://doi.org/10.1175/2011JAS3715.1.

— G. M. Heymsfield, P. D. Reasor, and S. R. Guimond, 2017: Concentric eyewall asymmetries in Hurricane Gonzalo (2014) observed by airborne radar. Mon. Wea. Rev., 145, 729-749, https://doi.org/10.1175/MWR-D-16-0175.1.

Fischer, M. S., R. F. Rogers, and P. D. Reasor, 2020: The rapid intensification and eyewall replacement cycles of Hurricane Irma (2017). Mon. Wea. Rev., 148, 981-1004, https://doi.org/ 10.1175/MWR-D-19-0185.1.

Gall, R., J. Tuttle, and P. Hildebrand, 1998: Small-scale spiral bands observed in Hurricanes Andrew, Hugo, and Erin. Mon. Wea. Rev., 126, 1749-1766, https://doi.org/10.1175/ 1520-0493(1998)126<1749:SSSBOI >2.0.CO;2.

Germano, M., 1986: A proposal for a redefinition of the turbulent stresses in the filtered Navier-Stokes equations. Phys. Fluids, 29, 2323-2324, https://doi.org/10.1063/1.865568.

Guimond, S. R., G. M. Heymsfield, and F. J. Turk, 2010: Multiscale observations of Hurricane Dennis (2005): The effects of hot towers on rapid intensification. J. Atmos. Sci., 67, 633-654, https://doi.org/10.1175/2009JAS3119.1.

_, L. Tian, G. M. Heymsfield, and S. J. Frasier, 2014: Wind retrieval algorithms for the IWRAP and HIWRAP airborne Doppler radars with applications to hurricanes. J. Atmos. Oceanic Technol., 31, 1189-1215, https://doi.org/10.1175/ JTECH-D-13-00140.1.

, G. M. Heymsfield, P. D. Reasor, and A. C. Didlake, 2016: The rapid intensification of Hurricane Karl (2010): New remote sensing observations of convective bursts from the Global Hawk platform. J. Atmos. Sci., 73, 3617-3639, https://doi.org/ 10.1175/JAS-D-16-0026.1.

_ J. A. Zhang, J. Sapp, and S. J. Frasier, 2018: Coherent turbulence in the boundary layer of Hurricane Rita (2005) during an eyewall replacement cycle. J. Atmos. Sci., 75, 3071-3093, https://doi.org/10.1175/JAS-D-17-0347.1.

Guinn, T. A., and W. H. Schubert, 1993: Hurricane spiral bands. J. Atmos. Sci., 50, 3380-3403, https://doi.org/10.1175/15200469(1993)050<3380:HSB >2.0.CO;2.

Kuo, H.-C., W. H. Schubert, C.-L. Tsai, and Y.-F. Kuo, 2008: Vortex interactions and the barotropic aspects of concentric eyewall formation. Mon. Wea. Rev., 136, 5183-5198, https:// doi.org/10.1175/2008MWR2378.1.

Kurihara, Y., 1976: On the development of spiral bands in a tropical cyclone. J. Atmos. Sci., 33, 940-958, https://doi.org/ 10.1175/1520-0469(1976)033<0940:OTDOSB > 2.0.CO;2.

Leonard, A., 1975: Energy cascade in large-eddy simulations of turbulent fluid flows. Adv. Geophys., 18, 237-248, https:// doi.org/10.1016/S0065-2687(08)60464-1.

Li, L., and Coauthors, 2016: The NASA High-altitude Imaging Wind and Rain Airborne Profiler. IEEE Trans. Geosci. Remote Sens., 54, 298-310, https://doi.org/10.1109/TGRS.2015.2456501.

Lilly, D. K., 1967: The representation of small-scale turbulence in numerical simulation experiments. Proc. IBM Scientific Symp. on Environmental Sciences, Yorktown Heights, NY, IBM, 195-210.

Macdonald, N. J., 1968: The evidence for the existence of Rossbylike waves in the hurricane vortex. Tellus, 20, 138-150, https:// doi.org/10.3402/tellusa.v20i1.9993.

Möller, J. D., and M. T. Montgomery, 2000: Tropical cyclone evolution via potential vorticity anomalies in a three-dimensional balance model. J. Atmos. Sci., 57, 3366-3387, https://doi.org/ 10.1175/1520-0469(2000)057<3366:TCEVPV>2.0.CO;2. 
Montgomery, M. T., and R. J. Kallenbach, 1997: A theory for vortex Rossby-waves and its application to spiral bands and intensity changes in hurricanes. Quart. J. Roy. Meteor. Soc., 123, 435-465, https://doi.org/10.1002/qj.49712353810.

, and J. Enagonio, 1998: Tropical cyclogenesis via convectively forced vortex Rossby waves in a three-dimensional quasigeostrophic model. J. Atmos. Sci., 55, 3176-3207, https://doi.org/10.1175/1520-0469(1998)055<3176:TCVCFV> 2.0.CO;2.

Moon, Y., and D. S. Nolan, 2015: Spiral rainbands in a numerical simulation of Hurricane Bill (2009). Part II: Propagation of inner rainbands. J. Atmos. Sci., 72, 191-215, https://doi.org/ 10.1175/JAS-D-14-0056.1.

Morrison, I., S. Businger, F. Marks, P. Dodge, and J. A. Businger, 2005: An observational case for the prevalence of roll vortices in the hurricane boundary layer. J. Atmos. Sci., 62, 2662-2673, https://doi.org/10.1175/JAS3508.1.

Qiu, X., Z.-M. Tan, and Q. Xiao, 2010: The roles of vortex Rossby waves in hurricane secondary eyewall formation. Mon. Wea. Rev., 138, 2092-2109, https://doi.org/10.1175/ 2010MWR3161.1.

Reasor, P. D., M. T. Montgomery, F. D. Marks Jr., and J. F. Gamache, 2000: Low-wavenumber structure and evolution of the hurricane inner core observed by airborne dual-Doppler radar. Mon. Wea. Rev., 128, 1653-1680, https://doi.org/ 10.1175/1520-0493(2000)128<1653:LWSAEO > 2.0.CO;2.

Schubert, W. H., M. T. Montgomery, R. K. Taft, T. A. Guinn, S. R. Fulton, J. P. Kossin, and J. P. Edwards, 1999: Polygonal eyewalls, asymmetric eye contraction, and potential vorticity mixing in hurricanes. J. Atmos. Sci., 56, 1197-1223,
https://doi.org/10.1175/1520-0469(1999)056<1197:PEAECA > 2.0.CO;2.

Smagorinsky, J., 1963: General circulation experiments with the primitive equations: I. The basic experiment. Mon. Wea. Rev., 91, 99-164, https://doi.org/10.1175/1520-0493(1963) 091<0099:GCEWTP>2.3.CO;2.

Stewart, S., 2017: Tropical cyclone report: Hurricane Matthew 28 September-9 October 2016. National Hurricane Center Rep., 96 pp., https://www.nhc.noaa.gov/data/tcr/AL142016_Matthew.pdf.

Stull, R., 1988: An Introduction to Boundary Layer Meteorology. Kluwer Academic, $666 \mathrm{pp}$.

Sullivan, P. P., T. W. Horst, D. Lenschow, C.-H. Moeng, and J. C. Weil, 2003: Structure of subfilter-scale fluxes in the atmospheric surface layer with application to large-eddy simulation modelling. J. Fluid Mech., 482, 101-139, https://doi.org/ 10.1017/S0022112003004099.

Terwey, W. D., and M. T. Montgomery, 2008: Secondary eyewall formation in two idealized, full-physics modeled hurricanes. J. Geophys. Res., 113, D12112, https://doi.org/ 10.1029/2007JD008897.

Willoughby, H. E., 1978: A possible mechanism for the formation of hurricane rainbands. J. Atmos. Sci., 35, 838-848, https://doi.org/ 10.1175/1520-0469(1978)035<0838:APMFTF $>2.0$.CO;2.

__ and P. G. Black, 1996: Hurricane Andrew in Florida: Dynamics of a disaster. Bull. Amer. Meteor. Soc., 77, 543-549, https:/doi.org/ 10.1175/1520-0477(1996)077<0543:HAIFDO > 2.0.CO;2.

_ J J. A. Clos, and M. G. Shoreibah, 1982: Concentric eye walls, secondary wind maxima, and the evolution of the hurricane vortex. J. Atmos. Sci., 39, 395-411, https://doi.org/10.1175/ 1520-0469(1982)039<0395:CEWSWM>2.0.CO;2. 